\title{
Analysis of Light Curves of Eclipsing Systems with Exoplanets: HD 189733
}

\author{
M. K. Abubekerov, N. Yu. Gostev, and A. M. Cherepashchuk \\ Sternberg Astronomical Institute, Moscow State University \\ marat@sai.msu.ru, ngostev@mail.ru, cher@sai.msu.ru
}

\begin{abstract}
High-accuracy multicolor light curves of the binary system HD 189733, which contains an exoplanet, are analyzed. We have determined the radii of the star and the planet in the binary system, as well as the orbital inclination. The limb-darkening coefficients of the stellar disk were obtained in 10 filters in the wavelength interval $\lambda \lambda=5500-10500 \AA$. The uncertainties of the fitted parameters were estimated using the differential-correction and confidence-area methods. The wavelength dependence of the limb-darkening coefficients is compared to the corresponding theoretical function for a model thin stellar atmosphere. We confirm the wavelength dependence of the exoplanet's radius found by Pont et al. [6] (at the $1 \sigma$ level). The exoplanet radius increases with decreasing wavelength, which seems to argue for the presence of an atmosphere around the planet.
\end{abstract}

\section{INTRODUCTION}

The results of fitting high-accuracy multicolor light curves for the star HD 209458, which is eclipsed by an exoplanet, were provided in [1]. There was a significant deviation between the observed and theoretical limb-darkening coefficients, even though the most conservative values were used to estimate the "external" uncertainties in the model parameters [2, 3]. This confirms the discrepancy between observations and the theory of thin stellar atmospheres found in [4]. It is important to analyze limb darkening across other stellar disks that are eclipsed by their exoplanets to further study this effect. Pont et al. [5, 6] obtained high-accuracy multicolor light curves of HD 189733, which is eclipsed by an exoplanet, and examined the spotted disk of the star. Pont et al. [5, 6] indicated that the exoplanet could have an atmosphere. The purpose of the current paper is to study the limb darkening of the star in the eclipsing system HD 189733 as a function of wavelength in detail using the data obtained in [5]. We analyze in detail the effect of uncertainties in the parameters determined using different methods to fit the eclipsing light curves, as in [1].

\section{FITTING METHOD}

The method used to fit transit light curves observed in eclipsing binary systems containing exoplanets is described in detail in [1], and we will only consider the main concepts of the method here. We used a model consisting of two spherical stars in circular orbits, without any reflection effect or ellipsoidal effect. There are no spots on the surfaces of the stars. If the masses of a star and planet are $m_{s}=0.825 M_{\odot}$ and $m_{p}=1.15 M_{\text {Jup }}$ [7], 
the mean relative radius of the Roche lobe for the planet calculated using the formula of Eggleton [16] is $R_{R} / a=0.0531$, where $a$ is the radius of the relative orbit of the system.

Since the relative radius of the planet is $r_{p}=R_{p} / a \simeq 0.0175$ (see below), the planet fills its Roche lobe only to the degree $\mu_{p} \simeq 0.33$, which is considerably less than 0.5 . Therefore, a spherical approximation is quite satisfactory for the planet (neglecting flattening of the planet due to its axial rotation). The same can be said for the optical star.

The amplitude of brightness changes due to the reflection effect in the optical should be less than $10^{-5}$ magnitude at eclipse phases, which is negligible [1]. A weak wavelength dependance of the radius of the exoplanet in HD 189733 was suggested in [6], which could be indicative that the planet has an atmosphere. The refraction of the stellar light in the exoplanets atmosphere can distort the light curve when the star is eclipsed by the exoplanet. The effects of light refraction in eclipsing binary systems were calculated by Kudzei [8]. These effects should result in small humps (brightenings) in the light curve before and after the eclipse, as well as in the middle of the eclipse. Since there are no such humps in the light curves of HD 189733, refraction effects in these light curves can be assumed to be negligible.

When the star is eclipsed by its exoplanet, the light curve could be affected by gravitational microlensing. Such effects were studied in [9]. Microlensing was shown to be significant only for the stars with exoplanets whose orbital sizes exceed 10 AU (with corresponding orbital periods of $P>10 \mathrm{yr}$ ). Therefore, our model with two spherical components is quite applicable to the light curves of HD 189733.

We calculated the light curve using linear and quadratic limb-darkening laws to describe the brightness distribution across the stellar disk:

$$
\begin{gathered}
I(\rho)=I_{0}\left(1-x+x \sqrt{1-\frac{\rho^{2}}{r_{s}^{2}}}\right), \\
I(\rho)=I_{0}\left(1-x\left(1-\sqrt{1-\frac{\rho^{2}}{r_{s}^{2}}}\right)-y\left(1-\sqrt{1-\frac{\rho^{2}}{r_{s}^{2}}}\right)^{2}\right) .
\end{gathered}
$$

Here, $\rho$ is the polar distance from the center of the stellar disk, $I_{0}$ the brightness at the center of the disk, $r_{s}$ the radius of the stellar disk in radii of the relative orbit, and $\mathrm{x}$ the limb-darkening coefficient. The brightness at the center of the planetary disk, and accordingly the brightness at any point of this disk, are assumed to be zero. The planet eclipses the star when the orbital phase is $\theta=\pi$. The orbit is taken to be circular, and the radius of the relative orbit $a$ to be unity. The model does not include third light. The radius of the planet in relative orbital radii is denoted $r_{p}$. The desired parameters of our model are the radius of the star $r_{s}$, the radius of the planet $r_{p}$, the inclination of the orbit to the plane of the sky $i$, and the limb-darkening coefficient $x$; if the limb darkening is described by the quadratic function, the parameters also include the coefficient $y$.

The total light of the system is taken to be unity, and the observed brightness values to be normally distrubuted. The rms deviations of the observed brightnesses $\sigma$ are assumed to be known.

To search for the optimal (central) values of the model parameters and generate their confidence intervals, we used a residual equal to the sum of the squared differences between 
the observed and theoretical brightnesses (as functions of the desired parameters), divided by the dispersions of the observed brightnesses (the squares of the rms deviations $\sigma$ ). If amodel fits the observational data, this residual is distributed according to a $\chi_{M}^{2}$ law for the exact parameter values, where $M$ is the number of observational points. This residual (which is a convex function of the parameters) achieves its minimum for certain parameters values $r_{s}^{c}, r_{p}^{c}, i^{c}, x^{c}, y^{c}$, referred to as the central (or optimal) parameters. When the model fits the observational data, the distribution of the central parameter values can be considered to be normal in some vicinity of their mean values, if we neglect nonlinearity of the parameter-dependent brightness in this neighbourhood. This is justified when $\sigma$ is small and the number of observational points $M$ is large.

The central parameter values are random values distributed normally in this approximation. They can be used as a statistic in the differential-correction method or Monte-Carlo method (which, in a sense, is equivalent to the differential-correctionmethod [10, 11]). The error intervals for the parameters are chosen to be centered on the optimal values of the corresponding parameters; their half-widths are equal to the mean square estimates of the rms deviations of the current parameters $\sigma_{\text {est }}$ from their central values, multiplied by a coefficient corresponding to the chosen confidence level $\gamma(1 \sigma, 2 \sigma, 3 \sigma$, etc.) [11. In this case, the model is considered to be perfectly valid; if we have more than one parameter, each individual parameter is characterized by its individual confidence interval, which encompasses the true value of that parameter with a given probability $\gamma$ (independent of whether or not the true values of the other fitted parameters fall into their confidence intervals). We also assume that we can neglect non-linearity of the function describing the dependence of the brightness on the parameters within approximately one rms deviation around the central parameters. Numerical experiments carried out for eclipsing systems show that the probability for all the true parameter values to simultaneously fall into their confidence intervals is a factor of $1.2 \div 1.5$ lower than the given confidence level $\gamma$ [10]. Since in the differential-correction method (or Monte-Carlo method) the obtained central parameter values are distributed statistically according to a normal law with the strict a priori assumption that the model is perfectly valid, and information on the dispersion of the observed brightnesses is not used here (the confidence intervals are constructed using the mean square estimates of the central parameters $\sigma_{\text {est }}^{2}$ rather than the dispersions of their central values), the uncertainties of the parameters in the differentialcorrection method (or Monte-Carlo method) are internal uncertainties. As a rule, these are substantially underestimated. Popper [12] indicated that this underestimation can reach a factor of three to five for eclipsing systems.

In view of the above, we also used the confidencearea method to estimate the parameter uncertainties [13]. The advantage of this method is that the obtained confidence intervals for the parameters ensure that the exact values of all the parameters simultaneous fall into the corresponding confidence area (in a multidimensional parametric space) with the given probability $\gamma$. The probability that the exact value of an individual parameter falls into the corresponding confidence interval is greater than the given probability $\gamma$. In addition, the probability for the exact solution (a combination of all fitted parameters) to be covered simultaneously by all the confidence intervals is also greater than $\gamma$, since the volume of the parallelepiped in which the confidence area $D$ is inscribed exceeds the volume of this area. 
Therefore, the confidence-area method yields more conservative external estimates of the parameter uncertainties, which are a factor of a few greater than the error estimates obtained using the differentialcorrection method orMonte-Carlo method.

We will use either a $\chi_{P}^{2}$ or a $\chi_{M}^{2}$ distribution to find the confidence area $D$ [10, 11]. We will use the difference between the residual distributed according to the $\chi_{M}^{2}$ law (M is the number of observational points) and the minimal value achieved with the central parameter values as the $\chi_{P}^{2}$ statistic, where $P$ is the number of fitted parameters. If we neglect the non-linearity in the dependence of the brightness on the parameters (which is justified when there are many observational points $M$ ), these differences can be considered to be distributed according to the $\chi_{P}^{2}$ law for the exact parameter values, where $P=4$ with linear limb darkening and $P=5$ with quadratic limb darkening.

When using statistics distributed according to $\chi_{M}^{2}$ or $\chi_{P}^{2}$, the confidence area $D$ is taken to be a multidimensional set in the parameter space, for which the specified statistics are less than the quantile corresponding to the given confidence level $\gamma$. The probability for $D$ to encompass the combined exact parameter values is then $\gamma$. The confidence intervals (uncertainties) for the parameters corresponding to a given confidence level $\gamma$ are the projections of the sides of the $P$-dimensional parallelepiped (in which the confidence area $D$ is inscribed) onto the coordinate axes in the parameter space [10]. As was noted above, this method for constructing the confidence intervals ensures that they will simultaneously encompass the exact values of all the parameters with the given probability $\gamma$ (or higher). Therefore, when specifying the confidence intervals (uncertainties) for the parameters, we should bear in mind that the adopted $\gamma$ (for the differential-correction or Monte- Carlo method) is equal to the probability for the exact value of each fixed parameter to be encompassed by the corresponding confidence interval; at the same time, the probability for the exact solution to be encompassed simultaneously in the all confidence intervals is less than $\gamma$. When we use the confidence area method, the probability for the exact value of a fixed parameter to be included in each individual confidence interval is greater than the adopted $\gamma$, whereas the probability that the confidence area $D$ encompasses the combined exact values of all the parameters is $\gamma$. Therefore, the adopted confidence level $\gamma$ specifies each of the individual confidence levels in the differential-correction method and Monte-Carlo method, and is related to the total confidence area $D$ in the confidence-area method.

Similar to the differential-correction method, using the $\chi_{P}^{2}$ statistic assumes that a model is perfectly valid, and that the obtained asymptotic confidence area $D$ never degenerates into an empty set. The confidence area $D$ does not depend on the sample size, and the probability $\gamma$ can be specified for an area $D$ that includes the exact solution (this probability is referred to as the confidence level). Since it is strictly assumed that the model is perfectly valid (the residuals are minimized over the parameters), the parameter uncertainties obtained using the $\chi_{P}^{2}$ statistic can also be considered to be internal, though these are more conservative than the uncertainties obtained using the differentialcorrection or Monte-Carlo method. We must bear in mind, however, that the dispersions of the observational data are used to construct the $\chi_{P}^{2}$ statistic; this is a significant difference between the use of the $\chi_{P}^{2}$ distribution and the differential-correction method. This difference can also be qualitatively significant if the rms dispersion with unit weighting (distributed according to a reduced $\chi^{2}$ law) differs significantly from unity; that is, when 
there is a basis to think that the model does not fit the observational data well, or a strong correlation is suspected in the observational data. The parameter uncertainties obtained using the $\chi_{P}^{2}$ distribution can also be considered external, since the dispersions of the external observational data are used here.

The most conservative "external" parameter uncertainties are obtained using the $\chi_{M}^{2}$ statistic, which does not assume the model to be perfectly valid and uses the dispersions of the observational data. It is also important that the same residual (the $\chi_{M}^{2}$ statistic) is used both to search for the central parameter values and to find their confidence intervals. However, the confidence set $D$ can be empty for certain significance levels $\alpha$ when using the $\chi_{M}^{2}$ statistic. As a result of fitting the observations, only a less strong statement can be made concerning the true parameter values, since we did not assume by default that the model is perfectly valid when using the $\chi_{M}^{2}$ statistic; namely, we can only indicate the probability (the significance level $\alpha$ ) that wemistakenly reject the hypothesis that the combined true parameter values belong to a certain area $D$ (rather than, strictly speaking, the probability $\gamma$ with which the confidence area $D$ encompasses this combination of parameters). If we suppose that the model fits the observational data, the probability for the area $D$ to encompass the exact values of all the parameters is $\gamma=1-\alpha$, where the probabilty $\gamma=1$ is referred to as the confidence level for the confidence area $D$. If, however, the model does not fit the observational data, this may mean that we make a second-order error in accepting the model (i.e., the model is false, but is accepted according to the statistical criterion). In this case, the equality $\gamma=1-\alpha$ is only approximate for the confidence area.

However, we must remember that, in most cases, we are dealing with a specific realization (a sample) of a light curve rather than the entire observed light curve (a general population). Therefore, difficulties that arise when trying to demonstrate the adequacy of a model may be associated with considerable statistical deviations of a specific observational data, rather than shortcomings of the model itself. A light curve obtained at another epoch may match the model adequately, with no difficulties arising when fitting the observations. Therefore, strictly speaking, a specific realization used in fitting does not necessarily imply that the model is inadequate; it implies only that the available observational data are not sufficient to ascertain whether the model is adequate or not. It is this that enables us to estimate the model parameters and their uncertainties even when the reduced $\chi^{2}, \chi_{\text {red }}^{2}$, is significantly greater than unity.

It follows from these considerations that searches for model parameters and their uncertainties in such problems should be accompanied by testing of the model adequacy. The $\chi_{M}^{2}$ statistic can be used to test the adequacy of a model and verify how it fits the observational data (since the model is not assumed to be perfectly valid). Here, we must bear in mind that we accept the model according to a statistical criterion not because it is perfectly valid, but because there is no reason to reject it.

We can judge how well the model fits the obesrvational data (whether the model is adequate enough) by estimating the significance level $\alpha=\alpha_{0}$ starting from which the model can be rejected (the value $\alpha_{0}$ is called the critical value of the significance level). The higher the critical value $\alpha_{0}$, the greater the probability that we make a first-order error when rejecting a model (that is, the weaker the basis for us to reject the model). The critical significance level $\alpha_{0}$ is associated with the reduced $\chi^{2}$ for the minimal residual, 
$\chi_{\text {red }}^{2}=\frac{\left(\chi_{M-P}^{2}\right)_{\text {min }}}{M-P}$, which decreases with increasing $\alpha_{0}[11$.

Strictly speaking, the residual minimized over nonlinear parameters in nonlinear parametric problems is not distributed according to a $\chi_{M-P}^{2}$ law, and only asymptotically approaches this law as $M \rightarrow \infty$ [13]. Therefore, the criterion for an adequate model that the $\chi_{\text {red }}^{2}$ red is close to unity can be used only when the number of observational points $M$ is sufficiently large. It was indicated in [11] that, if $\chi_{\text {red }}^{2}=1$, the critical significance level is $\alpha_{0} \simeq 50 \%$, which corresponds to a very good model. In fact, we have the possibility in this case (when choosing a corresponding significance level $\alpha$ ) to make up to $50 \%$ first-order errors in fitting if we reject the model; that is, we can err in every second case in rejecting the model. Hence, we have no reason to reject the model, and it can be accepted. When $\chi_{\text {red }}^{2}$ exceeds unity, the corresponding critical significance level is $\alpha_{0}<50 \%$. In this case, we do not make a large number of firstorder errors in rejecting the model; i.e., we are correct in most cases when we reject the model, and we therefore have a basis to reject the model. If, however, we obtain $\chi_{\text {red }}^{2}<1$, the corresponding significance level is $\alpha_{0}>50 \%$. When the observational data are normally distributed, this situation is very unlikely, even for a model that is perfectly valid (see, for example, [1]); therefore, the value $\chi_{\text {red }}^{2}<1$, and the corresponding value $\alpha_{0}>50 \%$, can indicate the presence of a correlation in the input observational data, which may include not only random but also systematic errors.

Finally, let us illustrate again why it is necessary to verify the model adequacy when deriving parameters from fits. An independent way of verifying the model adequacy follows from the fact that the number of observational points $M$ usually greatly exceeds the number of parameters $P$, i.e., as a rule, the problem is highly overdetermined. Strictly speaking, one should have an exact light curve corresponding to the true parameter values to estimate their uncertainties. However, an exact light curve (corresponding to the perfect parameter values) is not available in reality; we have only an observed light curve, subject to observational uncertainties. The question thus arises of how well the observed light curvematches the perfect light curve. In other words, before finding the solution from a fit, we must answer the question of whether we have a sufficient basis to substitute the observed light curve for an exact light curve that is unknown a priori.

We can answer this fundamentally important question after checking the models adequacy. If $\chi_{\text {red }}^{2} \simeq 1$, that is, $\alpha_{0} \simeq 50 \%$, there is no reason to reject the model, and the model can be accepted. An imagined perfectly exact light curve would optimally pass through the observational points of the real light curve, and we would have a firm basis to substitute the observed light curve for the perfectly exact light curve. The obtained values of the model parameters are close to their true values. Moreover, since the observed light curve optimally matches the perfectly exact curve and the contribution of systematic errors to the "exact" curve is negligible, we can obtain reliable uncertainty estimates that encompass the true parameter values with the given probability $\gamma$. We can estimate the parameter uncertainties using various methods, depending on the strictness with which we wish to judge the solution. These include the differential-correction or Monte-Carlo methods, which enable us to obtain "internal" uncertainties, the confidence-area method based on the $\chi_{M}^{2}$ statistic, which yields "external" uncertainties, or the confidence-area method based on the $\chi_{P}^{2}$ statistic (the intermediate case).

However, if we obtain $\chi_{\text {red }}^{2}>1$ and, correspondingly, $\alpha_{0}<50 \%$, we cannot make an unambiguous judgement about whether or not to accept the model. The modelmay prove 
to be "bad" since we can adopt for the model only a significance level $\alpha$ for which the probability to make a first-order error is small; i.e., we must only rarely be mistaken when rejecting the model. In this case, the statistical criterion suggests that the model should be rejected, and we construct a new and more perfect model. It is often the case in practice that $\chi_{\text {red }}^{2}>1$ and the corresponding critical significance level is $\alpha_{0}<10 \%$. In this case, if we accept the model, we must remember that the obtained parameters and their confidence intervals are subject to systematic errors due to either the fact that the perfectly exact light curve does not optimally pass through the observed light curve, or the presence of a strong correlation in the observational data (note that, in this case, we cannot unambiguously judge the systematic error to be due to a correlation in the observational data, in contrast to the case when $\alpha_{0}>50 \%$ ). Of course, the obtained model parameters and their uncertainties will be less reliable in these cases. The questions raised above are considered in more detail elsewhere [1, 10, 11, 13.

\section{OBSERVATIONAL DATA}

We analyze here a multicolor transit light curve of the binary system HD 189733, which contains an exoplanet [5]. The light curves were obtained with the Hubble Space Telescope during three observing runs, on May 22 and 26 and July 14, 2006, each lasting five orbital turns of the space observatory.

The observations of HD 189733 were carried out using the Advanced Camera for Surveys (ACS) in the HRC mode. In total, ten light curves with eclipses were obtained in the ranges $\lambda \lambda=5500-6000 \AA$, $6000-6500 \AA$, $6500-7000 \AA$, $7000-7500 \AA$, $7500-8000 \AA$, $8000-8500 \AA, 8500-9000 \AA, 9000-9500 \AA, 9500-10000 \AA, 10000-10500 \AA$. More detailed information about the observational data is available in [5]. When analyzing the light curves, we adopted the central wavelengths $\lambda \lambda=5750 \AA, 6250 \AA, 6750 \AA, 7250 \AA$, $7750 \AA$, $8250 \AA$, $8750 \AA$, $9250 \AA, 9750 \AA, 10250 \AA$.

Each light curve contains 675 individual brightness estimates. Figure 1 shows the observed light curve in the wavelength range $\lambda=5500-6000 \AA$ (central wavelength $\lambda=5750 \stackrel{\AA}{A})$.

We can see from Fig.1 that spots on the surface of the star [5] give rise to considerable brightness changes at eclipse phases. There is an appreciable shift in brightness between the right and left parts of the light curve due to either a spot on the surface of the star or systematic errors in the observations. These features required careful analysis and some corrections to the light curve.

We assumed that the observational uncertainties could be described by a normal distribution. The dispersions $\sigma^{2}$ of the individual points in the light curve were assumed to be the same for all points in the light-curve section used for the fitting. The dispersions were determined by averaging the squared differences between the observed points outside eclipse and themean brightness outside eclipse, using the left and right branches of the light curve both together and separately. We ascribed the resulting values of $\sigma$ as the rms deviations of the individual brightness measurements when fitting the corresponding part of the light curve.

We excluded part of the first observing run outside the eclipse at phases $\theta<160^{\circ}$, where an obvious systematic shift was observed relative to all other brightness measure- 
Table 1: rms deviations and the brightness outside eclipse for the light curves

\begin{tabular}{c|c|c|c|c|c|c|c|c|c}
$\lambda(\AA)$ & $\mathrm{E}_{\text {both }}$ & $\sigma_{\text {both }}$ & $M_{\text {both }}$ & $L_{\text {left }}$ & $\sigma_{\text {left }}$ & $M_{\text {left }}$ & $L_{\text {right }}$ & $\sigma_{\text {right }}$ & $M_{\text {right }}$ \\
\hline 5750 & 0.99998229 & 0.00007672 & 146 & 0.99997273 & 0.000078 & 79 & 0.99999736 & 0.000065 & 67 \\
6250 & 0.99997042 & 0.0003168 & 146 & 0.99994661 & 0.00035 & 79 & 1.0000079 & 0.00026 & 67 \\
6750 & 0.99994702 & 0.0002085 & 145 & 0.9999172 & 0.00022 & 78 & 0.99999414 & 0.00017 & 67 \\
7250 & 0.99996076 & 0.0001496 & 145 & 0.99993531 & 0.00016 & 78 & 1.000001 & 0.0001 & 67 \\
7750 & 0.99997562 & 0.0001421 & 146 & 0.99996303 & 0.00014 & 79 & 0.99999546 & 0.00014 & 67 \\
8250 & 0.99999351 & 0.0001321 & 147 & 0.99999245 & 0.00013 & 80 & 0.99999517 & 0.00013 & 67 \\
8750 & 1.0000042 & 0.0001558 & 147 & 1.0000095 & 0.00016 & 80 & 0.9999958 & 0.00015 & 67 \\
9250 & 0.99999268 & 0.0001766 & 147 & 0.99999 & 0.00018 & 80 & 0.9999969 & 0.00017 & 67 \\
9750 & 1.0000026 & 0.0002123 & 145 & 1.0000063 & 0.00022 & 78 & 0.99999678 & 0.0002 & 67 \\
10250 & 1.0000438 & 0.0003353 & 148 & 1.0000711 & 0.00036 & 80 & 1.0000006 & 0.00028 & 68 \\
\hline
\end{tabular}

ments outside the eclipse.

The mean brightness $L$ outside the eclipse, rms deviations of the individual brightness measurements $\sigma$, and the number of observational points in the eclipse $M$ for each light curve (labeled "both" for the left and right branches together, "left" for the left branch alone, and "right" for the right branch alone) are given in Table. The accuracy of the observed light curve is $\sim 10^{-4}$, which is $\sim 1 \%$ of the eclipse depth.

The main feature of the observed eclipse light curves is the distortion at the minimum (at phases $180^{\circ}<\theta<190^{\circ}$ for the points from the first observing run), due to spots on the surface of the star [5]. Moreover, even if this effect is excluded from consideration, the light curve remains appreciably distorted near the minimum at $\theta \sim 180^{\circ}$. Therefore, in addition to fitting using all the points in the light curve, we also analyzed the left (descending) branch of the light curve without $\theta>180^{\circ}$ and the right (ascending) branch without points $\theta<180^{\circ}$.

The resulting light curves that were analyzed are presented in 2. For ease of viewing, the zero points of the light curves for different $\lambda$ are shifted relative to each other. The theoretical light curves and the corresponding residual curves calculated using the best-fit model with quadratic limb-darkening are shown in the same figure.

\section{FITTING OF THE LIGHT CURVES USING THE LINEAR LIMB- DARKENING LAW}

The parameters derived from the observed light curves are the radius of the star $r_{s}$, radius of the exoplanet $r_{p}$, orbital inclination $i$, and coefficient $x$ in the linear limbdarkening law. The HD 189733 system contains a star of spectral type K2V [7]. The orbital period was taken to be $P_{\text {orb }}=2^{d} .218581$ [5], the planet-to-star mass ratio to be $q=m_{p} / m_{s}=0.014$ [5], the orbit of the system to be circular, and the radius of the relative orbit to be unity. The residual was minimized simultaneously over all parameters. Observational points from the phase interval covering only the eclipsed part of the light curve were used (the numbers of such points $\mathrm{M}$ for corresponding parts of the light curves are given in Table ${ }_{\text {z }}$. The parts of the light curves outside the eclipse were not used in the fitting, because they were used to independently determine the rms deviations of the points in the light curves (Table ${ }_{\text {a }}$. 


\section{Differential-Correction Method}

The results of fitting of the left and right parts of the transit light curve and the light curve as a whole are given in Tables 2, 3, and 4, respectively. The central parameter values and their $2 \sigma$ uncertainties were obtained using the differential-correction method. Since we used a model that most often proved to be "bad" (see below), we must provide $2 \sigma$ parameter uncertainties, corresponding to $\gamma=95.5 \%$. Moreover, when we determine the parameter uncertainties using the differential-correctionmethod in a multiparametric model, the true value of each parameter falls into the corresponding error interval with the given probability regardless of whether the true values of the other parameters fall into their error intervals.

The question thus arises: what is the probability of all the parameters falling into their error intervals? It is natural that we want this probability to be at least at the specified confidence level. If we consider the combined central parameter values to be a $\mathrm{P}$-dimensional random value varying in the $\mathrm{P}$-dimensional parameter space $\beta_{1} \ldots \beta_{P}$, then, when the true value $\bar{\beta}_{i}$ of one parameter $\beta_{i}$ falls into the corresponding error interval, the point associated with the combined true values will fall into the area between the two (P-1)-dimensional hyperplanes $\beta_{i}=\beta_{i}^{c}-\kappa \sigma_{i}$ and $\beta_{i}=\beta_{i}^{c}+\kappa \sigma_{i}$ ( $\kappa$ is a coefficient corresponding to the chosen confidence level).

Therefore, the differential-correctionmethod is essentially equivalent to finding a Pdimensional confidence area of this type. When the true parameter values fall into their corresponding error intervals, the point associated with the combined true parameter values falls into the $\mathrm{P}$-dimensional parallelepiped centered on the point $\beta_{1}^{c}, \ldots, \beta_{P}^{c}$ and with sides of length $2 \kappa \sigma_{1}, \ldots, 2 \kappa \sigma_{P}$. It is obvious that the probability for the combined true values to fall into this parallelepiped will be less than the probability for the true parameter values to independently fall into their error intervals, that is, less than the given probability. To ensure that a point in the $\mathrm{P}$-dimensional parameter space corresponding to the combined true parameter values falls into the P-dimensional parallelepiped centered on the point $\beta_{1}^{c}, \ldots, \beta_{P}^{c}$ and with sides proportional to $\sigma_{1}, \ldots, \sigma_{P}$ with the given probability $\gamma$ (i.e., to achieve a situation where the true parameter values all fall simultaneously into their corresponding intervals with the given probability $\gamma$ ), we must choose the intervals (dimensions of the $\mathrm{P}$-dimensional parallelepiped) to be $\mathrm{k} \kappa \sigma_{i}$, where $\mathrm{k}>1$.

The probability for a $\mathrm{P}$-dimensional random point to fall into the $\mathrm{P}$-dimensional parallelepiped is equal to the $\mathrm{P}$-fold integral of the multidimensional density distribution over the specified parallelepiped. It is not only the rms deviations of the random values $\beta_{1}^{c}, \ldots, \beta_{P}^{c}$ that we need to know before defining the type of density function (and determining the integral of the function). If a model is a linear function of the parameters $\beta_{1}, \ldots, \beta_{P}$ (which have the Gaussiandistributed central values $\beta_{1}^{c}, \ldots, \beta_{P}^{c}$ ) [10]), this distribution function will be written where $K$ is a normalizing coefficient and the matrix $A$ is the inverse of the covariance matrix $\operatorname{cov}\left(\beta_{i}, \beta_{j}\right)$ 1

\footnotetext{
${ }^{1}$ The exponent in (3) is the difference between the residual functional as a function of the parameters and the minimum value of this functional [10, and each level surface of the function $\mathfrak{f}$ (which is a Pdimensional ellipsoid) bounds a confidence area obtainedwith a certain confidence level using the $\chi_{P}^{2}$ distribution.
} 


$$
\mathfrak{f}\left(\beta_{1}, \ldots, \beta_{P}\right)=K e^{\sum_{i, j=1}^{P} A_{i j}\left(\beta_{i}-\bar{\beta}_{i}\right)\left(\beta_{j}-\bar{\beta}_{j}\right)}
$$

Therefore, even if we use a linear model (or a linear approximation) to determine the probability for the true parameter values to jointly fall into their error intervals (or if we wish to solve for the coefficient $\mathrm{k}$ ), we must know the covariances of the central values. The same values of the rms deviations $\sigma_{1}, \ldots, \sigma_{P}$ can correspond to different joint probabilities (different coefficients $\mathrm{k}$ ) in different cases. In any case, however, $\mathrm{k} \kappa \sigma_{i}$ will be less than the corresponding projections of the confidence area obtained using the $\chi_{P}^{2}$ distribution for the given $\gamma^{1}$

For example, for $\gamma=0.955$.. (that is, with $\kappa=2$ ), the projection of the confidence area obtained using the $\chi_{P}^{2}$ distribution is $\Delta_{P}=3.12 \sigma$ for a four-parameter model and $\Delta_{P}=3.36 \sigma$ for a five-parameter model [10]. Hence, at the " $2 \sigma$ " $(\gamma=0.955 .$.$) confidence$ level, $\mathrm{k} \lesssim 1.56$ for a four-parameter linear model and $\mathrm{k} \lesssim 1.68$ for a fiveparameter linear model. Note that $\mathrm{k} \lesssim 2.17$ for a fourparameter model and $\mathrm{k} \lesssim 2.42$ for a five-parameter model at the 1 confidence level $(\gamma=0.68)$.

In addition to the parameter values, table.2, table 3 , table 4also contain the minimum reduced $\chi^{2}$ values $\chi_{\text {red }}^{2}=\left(\chi_{M-P}^{2}\right)_{\min } /(M-P)$, and the critical significance levels $\alpha_{0}$. As was already noted, the $\chi_{\text {red }}^{2}$ values can be used to test whether the model adequately fits the observational data. This indicates (Tables 2, 3, 4) that our model was "bad" in most cases. When fitting the right branch of the light curve (Table 3).

Fitting of the left branch of the light curve (Table 2) yielded $\chi_{\text {red }}^{2}<1$ for three of ten wavelengths, with the corresponding $\alpha_{0}>50 \%$. This suggests that systematic errors strongly affect the left branch of the light curve. As was noted above, it is these difficulties $\left(\left(\chi_{\text {red }}^{2}<1\right.\right.$ and $\left.\chi_{\text {red }}^{2}\right)$ with fitting the light curves of the HD 189733 system that force us to adopt the confidence level $\gamma=95.5 \%$ (which corresponds to $2 \sigma$ in the differentialcorrection method) rather than $68 \%$, indicative of "good" models. For the same reason, we were not able to construct "exact" confidence areas D using the $\chi_{M}^{2}$ distribution and to provide the most conservative estimates of the "external" parameter uncertainties for the right branch of the light curve (Table 3) and for the entire light curve (Table 4). We restricted our consideration to producing asymptotic confidence areas for the parameters using the $\chi_{P}^{2}$ statistic. We were able to construct the confidence areas for the left branch of the light curve using both the $\chi_{P}^{2}$ and the $\chi_{M}^{2}$ distributions.

\section{Confidence-Area Method}

We used the $\chi_{P}^{2}$ and $\chi_{M}^{2}$ distributions for the confidence-area method. We took $\gamma=$ $95.5 \%$, which corresponds to $2 \sigma$ for the differential-correction method, where $\sigma$ is the rms deviation. The $\chi_{M}^{2}$ statistic was used only for the left branch of the light curve. The fitting results for the left branch of the light curve obtained using the confidence-area method based on the $\chi_{M}^{2}$ statistic are given in Table 8 ,

\footnotetext{
${ }^{1}$ Since these projections are dimensions of the $P$-dimensional parallelepiped that are proportional to $\sigma_{1}, \ldots, \sigma_{P}$, the probability for them to encompass the true parameter values is greater than $\gamma$.
} 
Table 2: Fitting of the left branches of the observed light curves for HD 189733 [6] with the linear limb-darkening law. (Parameter uncertainties estimated using the differentialcorrection method are given at the $2 \sigma$ level. The two last columns give the reduced $\chi^{2}$ and the corresponding $\alpha_{0}$.)

\begin{tabular}{c|c|c|c|c|c|c|c|c|c|c}
$\lambda(\AA)$ & $r_{s}^{c}$ & $2 \sigma_{\text {est }}\left(r_{s}^{c}\right)$ & $r_{p}^{c}$ & $2 \sigma_{\text {est }}\left(r_{p}^{c}\right)$ & $i^{c}\left(^{\circ}\right)$ & $2 \sigma_{\text {est }}\left(i^{c}\right)\left(^{\circ}\right)$ & $x^{c}$ & $2 \sigma_{\text {est }}\left(x^{c}\right)$ & $\chi_{r e d}^{2}$ & $\alpha_{0}$ \\
\hline 5750 & 0.11184 & 0.00054 & 0.01760 & 0.00011 & 85.715 & 0.054 & 0.555 & 0.017 & 2.3457 & $1.9 \cdot 10^{-9}$ \\
6250 & 0.1130 & 0.0011 & 0.01790 & 0.00025 & 85.60 & 0.11 & 0.609 & 0.037 & 0.51644 & 0.99996 \\
6750 & 0.11185 & 0.00090 & 0.01765 & 0.00020 & 85.697 & 0.091 & 0.615 & 0.028 & 0.78673 & 0.956 \\
7250 & 0.11133 & 0.00073 & 0.01751 & 0.00016 & 85.754 & 0.073 & 0.590 & 0.022 & 0.96149 & 0.695 \\
7750 & 0.11172 & 0.00066 & 0.01758 & 0.00014 & 85.716 & 0.065 & 0.556 & 0.020 & 1.1113 & 0.345 \\
8250 & 0.11219 & 0.00073 & 0.01768 & 0.00015 & 85.693 & 0.072 & 0.540 & 0.023 & 1.5651 & 0.0031 \\
8750 & 0.11225 & 0.00072 & 0.01765 & 0.00015 & 85.682 & 0.071 & 0.519 & 0.023 & 1.0306 & 0.53 \\
9250 & 0.11186 & 0.00080 & 0.01756 & 0.00016 & 85.731 & 0.079 & 0.490 & 0.025 & 1.0506 & 0.48 \\
9750 & 0.1110 & 0.0010 & 0.01740 & 0.00021 & 85.79 & 0.10 & 0.479 & 0.033 & 1.1975 & 0.19 \\
10250 & 0.1117 & 0.0013 & 0.01755 & 0.00026 & 85.75 & 0.13 & 0.497 & 0.041 & 0.69904 & 0.99 \\
\hline
\end{tabular}

Table 3: Fitting of the right branches of the observed light curves for HD 189733 [6] with the linear limb-darkening law. (Parameter uncertainties estimated using the differentialcorrection method are given at the $2 \sigma$ level. The two last columns give the reduced $\chi^{2}$ and the corresponding $\alpha_{0}$.)

\begin{tabular}{|c|c|c|c|c|c|c|c|c|c|c|}
\hline$\lambda(\stackrel{\AA}{A})$ & $r_{s}^{c}$ & $2 \sigma_{\text {est }}\left(r_{s}^{c}\right)$ & $r_{p}^{c}$ & $2 \sigma_{\text {est }}\left(r_{p}^{c}\right)$ & $i^{c}\left(^{\circ}\right)$ & $2 \sigma_{\text {est }}\left(i^{c}\right)\left({ }^{\circ}\right)$ & $x^{c}$ & $2 \sigma_{\text {est }}\left(x^{c}\right)$ & $\chi_{\text {red }}^{2}$ & $\alpha_{0}$ \\
\hline 5750 & 0.11159 & 0.00069 & 0.01738 & 0.00015 & 85.793 & 0.072 & 0.578 & 0.023 & 4.2535 & 0 \\
\hline 6250 & 0.1124 & 0.0015 & 0.01761 & 0.00034 & 85.71 & 0.16 & 0.590 & 0.053 & 1.3380 & 0.075155 \\
\hline 6750 & 0.1118 & 0.0012 & 0.01746 & 0.00028 & 85.77 & 0.13 & 0.631 & 0.041 & 1.9672 & 0.000029131 \\
\hline 7250 & 0.11164 & 0.00085 & 0.01741 & 0.00019 & 85.791 & 0.091 & 0.617 & 0.028 & 2.6644 & $1.2811 \cdot 10^{-10}$ \\
\hline 7750 & 0.11173 & 0.00086 & 0.01740 & 0.00019 & 85.784 & 0.091 & 0.593 & 0.029 & 1.4333 & 0.030473 \\
\hline 8250 & 0.11177 & 0.00078 & 0.01742 & 0.00017 & 85.786 & 0.082 & 0.570 & 0.026 & 1.3065 & 0.098526 \\
\hline 8750 & 0.11185 & 0.00088 & 0.01738 & 0.00019 & 85.771 & 0.092 & 0.534 & 0.030 & 1.4180 & 0.035518 \\
\hline 9250 & 0.11104 & 0.00092 & 0.01725 & 0.00019 & 85.845 & 0.097 & 0.521 & 0.032 & 1.1435 & 0.31474 \\
\hline 9750 & 0.1108 & 0.0012 & 0.01727 & 0.00025 & 85.84 & 0.12 & 0.497 & 0.042 & 1.4684 & 0.021212 \\
\hline 10250 & 0.1110 & 0.0013 & 0.01726 & 0.00027 & 85.83 & 0.13 & 0.506 & 0.045 & 0.84083 & 0.89432 \\
\hline
\end{tabular}

Table 4: Joint fitting of the right and left branches of the observed light curves for HD 189733 [6] with the linear limbdarkening law. (Parameter uncertainties estimated using the differential-correctionmethod are given at the $2 \sigma$ level. The two last columns give the reduced $\chi^{2}$ and the corresponding $\left.\alpha_{0}\right)$.

\begin{tabular}{c|c|c|c|c|c|c|c|c|c|c}
$\lambda(\AA)$ & $r_{s}^{c}$ & $2 \sigma_{\text {est }}\left(r_{s}^{c}\right)$ & $r_{p}^{c}$ & $2 \sigma_{\text {est }}\left(r_{p}^{c}\right)$ & $i^{c}\left(^{\circ}\right)$ & $2 \sigma_{\text {est }}\left(i^{c}\right)\left({ }^{\circ}\right)$ & $x^{c}$ & $2 \sigma_{\text {est }}\left(x^{c}\right)$ & $\chi_{r e d}^{2}$ & $\alpha_{0}$ \\
\hline 5750 & 0.11192 & 0.00059 & 0.01755 & 0.00012 & 85.726 & 0.060 & 0.556 & 0.019 & 5.5168 & 0 \\
6250 & 0.1129 & 0.0011 & 0.01780 & 0.00024 & 85.64 & 0.11 & 0.600 & 0.036 & 1.0071 & 0.55 \\
6750 & 0.11204 & 0.00086 & 0.01761 & 0.00019 & 85.706 & 0.088 & 0.614 & 0.028 & 1.5321 & 0.00010 \\
7250 & 0.11171 & 0.00068 & 0.01753 & 0.00015 & 85.740 & 0.070 & 0.591 & 0.022 & 1.9306 & $6 \cdot 10^{-10}$ \\
7750 & 0.11196 & 0.00068 & 0.01756 & 0.00014 & 85.717 & 0.069 & 0.561 & 0.022 & 2.0916 & $10^{-12}$ \\
8250 & 0.11215 & 0.00066 & 0.01760 & 0.00014 & 85.715 & 0.067 & 0.545 & 0.022 & 2.2529 & $4 \cdot 10^{-15}$ \\
8750 & 0.11230 & 0.00073 & 0.01758 & 0.00015 & 85.695 & 0.073 & 0.515 & 0.024 & 2.0264 & $2 \cdot 10^{-11}$ \\
9250 & 0.11166 & 0.00070 & 0.01747 & 0.00014 & 85.758 & 0.071 & 0.494 & 0.023 & 1.4922 & 0.00029 \\
9750 & 0.11103 & 0.00080 & 0.01736 & 0.00016 & 85.804 & 0.080 & 0.482 & 0.026 & 1.3541 & 0.0063 \\
10250 & 0.11150 & 0.00096 & 0.01745 & 0.00020 & 85.765 & 0.097 & 0.493 & 0.032 & 0.79637 & 0.98 \\
\hline
\end{tabular}

The fitting results obtained using the confidencearea method based on the $\chi_{P}^{2}$ distribution are given in Tables 5, 6, 7. Here, the best-fit values of $r_{p}, r_{s}, i, x$ are given together with their uncertainties, characterized by the projections of the asymptotic confidence area $D$ in the four-parameter space onto the $r_{p}, r_{s}, i, x$ axes (the confidence intervals). To facilitate comparison with the uncertainties obtained in the differential-correction method (Tables 2 - 4), Tables 5 - 7 show the values $\Delta_{P}$, equal to half the confidence intervals. The tables for the confidencearea method present parameter values corresponding to the middles of the confidence intervals, rather than with the central parameter values. The probability that the exact value of each parameter $r_{p}, r_{s}, i, x$ is encompassed within the corresponding confidence interval exceeds $95.5 \%$. The probability that the exact solution 
(the combination of all parameters $\left.r_{p}, r_{s}, i, x\right)$ is encompassed within the asymptotic confidence area is close to the given probability $\gamma=95.5 \%$, since the number of points in the light curve is large $(M \gtrsim 70)$. The probability that the exact solution is simultaneously encompassed within all confidence intervals exceeds the given $\gamma=95.5 \%$.

Table 5: Fitting of the left branches of the observed light curves for HD 189733 [6] with the linear limb-darkening law. (Parameter uncertainties were estimated using the confidence-area method based on the $\chi_{P}^{2}$ distribution, with $\gamma=0.955$.)

\begin{tabular}{c|c|c|c|c|c|c|c|c}
$\lambda(\AA)$ & $r_{s}$ & $\Delta_{P}\left(r_{s}\right)$ & $r_{p}$ & $\Delta_{P}\left(r_{p}\right)$ & $i$ & $\Delta_{P}(i)$ & $x$ & $\Delta_{P}(x)$ \\
\hline 5750 & 0.11184 & 0.00055 & 0.01760 & 0.00011 & $85.715^{\circ}$ & $0.054^{\circ}$ & 0.555 & 0.017 \\
6250 & 0.1130 & 0.0025 & 0.01788 & 0.00055 & $85.61^{\circ}$ & $0.25^{\circ}$ & 0.607 & 0.080 \\
6750 & 0.1118 & 0.0016 & 0.01764 & 0.00035 & $85.70^{\circ}$ & $0.16^{\circ}$ & 0.614 & 0.049 \\
7250 & 0.1113 & 0.0012 & 0.01751 & 0.00025 & $85.76^{\circ}$ & $0.12^{\circ}$ & 0.590 & 0.035 \\
7750 & 0.11171 & 0.00094 & 0.01758 & 0.00020 & $85.717^{\circ}$ & $0.092^{\circ}$ & 0.556 & 0.029 \\
8250 & 0.11217 & 0.00091 & 0.01767 & 0.00019 & $85.695^{\circ}$ & $0.090^{\circ}$ & 0.540 & 0.029 \\
8750 & 0.1123 & 0.0011 & 0.01765 & 0.00022 & $85.68^{\circ}$ & $0.10^{\circ}$ & 0.518 & 0.034 \\
9250 & 0.1119 & 0.0012 & 0.01756 & 0.00025 & $85.73^{\circ}$ & $0.12^{\circ}$ & 0.490 & 0.040 \\
9750 & 0.1110 & 0.0015 & 0.01739 & 0.00029 & $85.79^{\circ}$ & $0.14^{\circ}$ & 0.477 & 0.046 \\
10250 & 0.1117 & 0.0026 & 0.01754 & 0.00053 & $85.75^{\circ}$ & $0.26^{\circ}$ & 0.493 & 0.082 \\
\hline
\end{tabular}

Table 6: Fitting of the right branches of the observed light curves for HD 189733 [6] with the linear limb-darkening law. (Parameter uncertainties were estimated using the confidence-area method based on the $\chi_{P}^{2}$ distribution, with $\gamma=0.955$.)

\begin{tabular}{c|c|c|c|c|c|c|c|c}
$\lambda(\AA)$ & $r_{s}$ & $\Delta_{P}\left(r_{s}\right)$ & $r_{p}$ & $\Delta_{P}\left(r_{p}\right)$ & $i$ & $\Delta_{P}(i)$ & $x$ & $\Delta_{P}(x)$ \\
\hline 5750 & 0.11159 & 0.00052 & 0.01738 & 0.00011 & $85.794^{\circ}$ & $0.055^{\circ}$ & 0.578 & 0.018 \\
6250 & 0.1124 & 0.0019 & 0.01760 & 0.00043 & $85.72^{\circ}$ & $0.20^{\circ}$ & 0.589 & 0.069 \\
6750 & 0.1118 & 0.0013 & 0.01745 & 0.00031 & $85.77^{\circ}$ & $0.14^{\circ}$ & 0.631 & 0.046 \\
7250 & 0.11164 & 0.00082 & 0.01741 & 0.00019 & $85.792^{\circ}$ & $0.089^{\circ}$ & 0.617 & 0.028 \\
7750 & 0.1117 & 0.0011 & 0.01739 & 0.00025 & $85.79^{\circ}$ & $0.12^{\circ}$ & 0.593 & 0.038 \\
8250 & 0.1118 & 0.0010 & 0.01742 & 0.00023 & $85.79^{\circ}$ & $0.11^{\circ}$ & 0.570 & 0.036 \\
8750 & 0.1118 & 0.0011 & 0.01737 & 0.00024 & $85.77^{\circ}$ & $0.12^{\circ}$ & 0.533 & 0.039 \\
9250 & 0.1110 & 0.0013 & 0.01725 & 0.00028 & $85.85^{\circ}$ & $0.14^{\circ}$ & 0.520 & 0.046 \\
9750 & 0.1108 & 0.0015 & 0.01726 & 0.00031 & $85.85^{\circ}$ & $0.16^{\circ}$ & 0.496 & 0.053 \\
10250 & 0.1110 & 0.0022 & 0.01725 & 0.00045 & $85.83^{\circ}$ & $0.23^{\circ}$ & 0.503 & 0.076 \\
\hline
\end{tabular}

\section{THE PLANET'S RADIUS AND LINEAR LIMB-DARKENING COEFFI- CIENT AS FUNCTIONS OF WAVELENGTH IN THE LINEAR LAW}

Our model for the system HD 189733 with a linear limb-darkening law proved to be "bad" for most wavelengths. This is not surprising, since the light curves trace spots on the surface of the star [6], which are not taken into account in the model. Only for the left branch of the light curve can ourmodel with linear limb-darkening be rejected for most wavelengths at a fairly high significance level, so that we can estimate the most 
Table 7: Joint fitting of the right and left branches of the observed light curves for HD 189733 [6] with the linear limbdarkening law. (Parameter uncertainties were estimated using the confidence-area method based on the $\chi_{P}^{2}$ distribution, with $\left.\gamma=0.955\right)$.

\begin{tabular}{c|c|c|c|c|c|c|c|c}
$\lambda(\AA)$ & $r_{s}$ & $\Delta_{P}\left(r_{s}\right)$ & $r_{p}$ & $\Delta_{P}\left(r_{p}\right)$ & $i$ & $\Delta_{P}(i)$ & $x$ & $\Delta_{P}(x)$ \\
\hline 5750 & 0.11192 & 0.00039 & 0.017552 & 0.000083 & $85.726^{\circ}$ & $0.040^{\circ}$ & 0.556 & 0.013 \\
6250 & 0.1129 & 0.0017 & 0.01780 & 0.00036 & $85.64^{\circ}$ & $0.17^{\circ}$ & 0.598 & 0.055 \\
6750 & 0.1120 & 0.0011 & 0.01761 & 0.00024 & $85.71^{\circ}$ & $0.11^{\circ}$ & 0.614 & 0.035 \\
7250 & 0.11170 & 0.00078 & 0.01752 & 0.00017 & $85.743^{\circ}$ & $0.080^{\circ}$ & 0.591 & 0.025 \\
7750 & 0.11194 & 0.00072 & 0.01755 & 0.00015 & $85.719^{\circ}$ & $0.072^{\circ}$ & 0.562 & 0.023 \\
8250 & 0.11215 & 0.00068 & 0.01760 & 0.00014 & $85.715^{\circ}$ & $0.069^{\circ}$ & 0.545 & 0.022 \\
8750 & 0.11229 & 0.00078 & 0.01758 & 0.00016 & $85.698^{\circ}$ & $0.078^{\circ}$ & 0.515 & 0.026 \\
9250 & 0.11166 & 0.00093 & 0.01747 & 0.00019 & $85.759^{\circ}$ & $0.095^{\circ}$ & 0.493 & 0.031 \\
9750 & 0.1110 & 0.0010 & 0.01736 & 0.00021 & $85.81^{\circ}$ & $0.10^{\circ}$ & 0.482 & 0.034 \\
10250 & 0.1115 & 0.0017 & 0.01745 & 0.00036 & $85.77^{\circ}$ & $0.18^{\circ}$ & 0.491 & 0.058 \\
\hline
\end{tabular}

Table 8: Fitting of the left branches of the observed light curves for HD 189733[6] with the linear limb-darkening law. (Parameter uncertainties were estimated using the confidencearea method based on the $\chi_{M}^{2}$ distribution, with $\left.\gamma=0.955\right)$.

\begin{tabular}{c|c|c|c|c|c|c|c|c}
$\lambda(\AA)$ & $r_{s}$ & $\Delta_{M}\left(r_{s}\right)$ & $r_{p}$ & $\Delta_{M}\left(r_{p}\right)$ & $i$ & $\Delta_{M}(i)$ & $x$ & $\Delta_{M}(x)$ \\
\hline 6250 & 0.1127 & 0.0066 & 0.0178 & 0.0014 & $85.67^{\circ}$ & $0.67^{\circ}$ & 0.59 & 0.22 \\
6750 & 0.1118 & 0.0034 & 0.01761 & 0.00074 & $85.71^{\circ}$ & $0.34^{\circ}$ & 0.61 & 0.10 \\
7250 & 0.1113 & 0.0020 & 0.01750 & 0.00043 & $85.76^{\circ}$ & $0.20^{\circ}$ & 0.589 & 0.061 \\
7750 & 0.1117 & 0.0013 & 0.01758 & 0.00027 & $85.72^{\circ}$ & $0.13^{\circ}$ & 0.556 & 0.040 \\
8750 & 0.1122 & 0.0017 & 0.01765 & 0.00035 & $85.69^{\circ}$ & $0.17^{\circ}$ & 0.517 & 0.055 \\
9250 & 0.1118 & 0.0019 & 0.01755 & 0.00039 & $85.74^{\circ}$ & $0.19^{\circ}$ & 0.488 & 0.061 \\
9750 & 0.1110 & 0.0016 & 0.01739 & 0.00032 & $85.79^{\circ}$ & $0.16^{\circ}$ & 0.477 & 0.051 \\
10250 & 0.1116 & 0.0059 & 0.0175 & 0.0012 & $85.78^{\circ}$ & $0.61^{\circ}$ & 0.47 & 0.19 \\
\hline
\end{tabular}

conservative "external" parameter uncertainties for $\gamma=95.5 \%$ using the $\chi_{M}^{2}$ distribution (Table 8).

The exoplanet-to-star radius ratio $r_{p} / r_{s}$ in the HD 189733 system was obtained in [6] as a function of wavelength. Our results based on our analysis of the complete light curves are given in Table 9, together with the results obtained in [6]. Since we are searching for the individual parameters $r_{p}$ and $r_{s}$, rather than their ratio $r_{p} / r_{s}$, the uncertainty in $r_{p} / r_{s}$ was obtained using the differential-correctionmethod as an uncertainty for a new parameter. The general formula for estimating the rms deviations when changing variables is given in [1]. In our case,

$$
\sigma\left(r_{p}^{c} / r_{s}^{c}\right)=\sqrt{\left(\frac{r_{p}^{c}}{\left(r_{s}^{c}\right)^{2}}\right)^{2} \sigma^{2}\left(r_{s}^{c}\right)-2 \operatorname{cov}\left(r_{p}^{c}, r_{s}^{c}\right)\left(\frac{r_{p}^{c}}{\left(r_{s}^{c}\right)^{2}}\right)\left(\frac{1}{r_{s}^{c}}\right)+\left(\frac{1}{r_{s}^{c}}\right)^{2} \sigma^{2}\left(r_{p}^{c}\right)}
$$

where $\operatorname{cov}(\cdot, \cdot)$ is the procedure for searching for the covariances of random values. Our uncertainties are larger than those of [6], where fits were obtained for the geometrical parameters $r_{s}, r_{p}, i$, with the limb-darkening coefficients being fixed. Since we fit the 
light curves allowing both the geometrical parameters and the limb-darkening coefficients to vary, the larger number of degrees of freedom in our model yields larger parameter uncertainties.

Figure 3 indicates a certain increase in the planet's radius with decreasing wavelength. This effect is significant at the $1 \sigma$ level, but not the $2 \sigma$ level. Our values of the exoplanet radius are systematically larger than those determined in [5] (by 0.3\%); this is due to the different normalization procedures applied in the analysis of the light curve and the fact that we searched for the limb-darkening coefficient together with other parameters, rather than fixing this coefficient.

Table 9: Comparison of the $r_{p} / r_{s}$ values as a function of wavelength obtained in this paper and in [6]. (The $1 \sigma$ uncertainties are given).

\begin{tabular}{c|c|c}
$\lambda(\AA)$ & & {$[6]$} \\
\hline 5750 & $0.156894 \pm 0.000281$ & $0.156903 \pm 0.000095$ \\
6250 & $0.157762 \pm 0.000658$ & $0.156744 \pm 0.000065$ \\
6750 & $0.157387 \pm 0.000458$ & $0.156552 \pm 0.000057$ \\
7250 & $0.157045 \pm 0.000341$ & $0.156388 \pm 0.000059$ \\
7750 & $0.156917 \pm 0.000358$ & $0.156501 \pm 0.000064$ \\
8250 & $0.156984 \pm 0.000348$ & $0.156210 \pm 0.000073$ \\
8750 & $0.156569 \pm 0.000388$ & $0.156147 \pm 0.000081$ \\
9250 & $0.156451 \pm 0.000391$ & $0.156120 \pm 0.000092$ \\
9750 & $0.156361 \pm 0.000463$ & $0.156097 \pm 0.000125$ \\
10250 & $0.156389 \pm 0.000611$ & $0.155716 \pm 0.000218$ \\
\hline
\end{tabular}

Table 10: Fitting of the left branches of the observed light curves for HD 189733 [6] with the quadratic limb-darkening law. (Parameter uncertainties estimated using the differential-correction method are given at the $2 \sigma$ level. The two last columns present the values of the reduced $\chi^{2}$ and the corresponding critical significance levels $\alpha_{0}$ ).

\begin{tabular}{|c|c|c|c|c|c|c|c|c|c|c|c|c|}
\hline$\lambda(\stackrel{\AA}{)}$ & $r_{s}^{c}$ & $2 \sigma_{\text {est }}\left(r_{s}^{c}\right)$ & $r_{p}^{c}$ & $2 \sigma_{\text {est }}\left(r_{p}^{c}\right)$ & $i^{c}\left({ }^{\circ}\right)$ & $2 \sigma_{\text {est }}\left(i^{c}\right)\left({ }^{\circ}\right)$ & $x_{1}^{c}$ & $2 \sigma_{\text {est }}\left(x_{1}^{c}\right)$ & $y_{1}^{c}$ & $2 \sigma_{\text {est }}\left(y_{1}^{c}\right)$ & $\chi_{\text {red }}^{2}$ & $\alpha_{0}$ \\
\hline 5750 & 0.11207 & 0.00083 & 0.01754 & 0.00022 & 85.721 & 0.077 & 0.44 & 0.16 & 0.18 & 0.26 & 2.3115 & $8 \cdot 10^{-9}$ \\
\hline 6250 & 0.1132 & 0.0020 & 0.01782 & 0.00050 & 85.62 & 0.17 & 0.50 & 0.37 & 0.16 & 0.60 & 0.52219 & 0.99997 \\
\hline 6750 & 0.1120 & 0.0014 & 0.01759 & 0.00035 & 85.70 & 0.12 & 0.52 & 0.26 & 0.14 & 0.42 & 0.79144 & 0.96 \\
\hline 7250 & 0.11144 & 0.00094 & 0.01748 & 0.00024 & 85.758 & 0.088 & 0.53 & 0.20 & 0.08 & 0.32 & 0.97038 & 0.70 \\
\hline 7750 & 0.11189 & 0.00091 & 0.01754 & 0.00023 & 85.720 & 0.083 & 0.47 & 0.20 & 0.13 & 0.30 & 1.1150 & 0.37 \\
\hline 8250 & 0.1125 & 0.0022 & 0.01754 & 0.00048 & 85.72 & 0.16 & 0.32 & 0.24 & 0.33 & 0.48 & 1.5210 & 0.0074 \\
\hline 8750 & 0.1123 & 0.0010 & 0.01757 & 0.00026 & 85.704 & 0.094 & 0.42 & 0.23 & 0.14 & 0.35 & 1.0401 & 0.54 \\
\hline 9250 & 0.1121 & 0.0013 & 0.01748 & 0.00036 & 85.74 & 0.12 & 0.34 & 0.26 & 0.23 & 0.41 & 1.0466 & 0.53 \\
\hline 9750 & 0.1112 & 0.0013 & 0.01737 & 0.00034 & 85.79 & 0.12 & 0.41 & 0.33 & 0.10 & 0.49 & 1.2103 & 0.198 \\
\hline 10250 & 0.1131 & 0.0044 & 0.0175 & 0.0012 & 85.71 & 0.29 & 0.05 & 0.43 & 0.65 & 0.45 & 0.66148 & 0.997 \\
\hline
\end{tabular}

Note that the difference between the exoplanet radius in HD 209458 obtained in the blue and the red does not seem to be significant [1]: $\overline{r_{p}}=0.0139 \pm 0.0003$ for $\bar{\lambda}=3750$

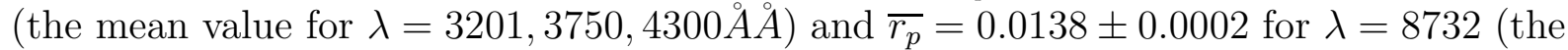
mean value for $\lambda=7755,8732,9708 \AA \AA$ ) ; the $2 \sigma$ uncertainties are indicated.

Let us now consider the limb-darkening coefficients in the linear limb-darkening law as a function of wavelength. Figures 4, 5, 6, 7) show the observed limbdarkening coefficients $x$ versus the wavelength $\lambda$, together with the theoretical linear dependences of the limbdarkening coefficients on in the photometric systems ugriz and UBVRIJ [2, 15]. The observed relation $x(\lambda)$ agrees qualitatively with the theoretical one: the observed $x(\lambda)$ 
Table 11: Fitting of the right branches of the observed light curves for HD 189733 [6] with the quadratic limb-darkening law. (Parameter uncertainties estimated using the differential-correction method are given at the $2 \sigma$ level. The two last columns present the values of the reduced $\chi^{2}$ and the corresponding critical significance levels $\alpha_{0}$ ).

\begin{tabular}{|c|c|c|c|c|c|c|c|c|c|c|c|c|}
\hline$\lambda(\stackrel{\AA}{A})$ & $r_{s}^{c}$ & $2 \sigma_{\text {est }}\left(r_{s}^{c}\right)$ & $r_{p}^{c}$ & $2 \sigma_{\text {est }}\left(r_{p}^{c}\right)$ & $i^{c}\left({ }^{\circ}\right)$ & $2 \sigma_{\text {est }}\left(i^{c}\right)\left({ }^{\circ}\right)$ & $x_{1}^{c}$ & $2 \sigma_{\text {est }}\left(x_{1}^{c}\right)$ & $y_{1}^{2}$ & $2 \sigma_{\text {est }}\left(y_{1}^{c}\right)$ & $\chi_{r e d}^{2}$ & $\alpha_{0}$ \\
\hline 5750 & 0.11094 & 0.00053 & 0.017531 & 0.000095 & 85.781 & 0.044 & 0.86 & 0.20 & -0.42 & 0.27 & 3.7576 & 0 \\
\hline 6250 & 0.1113 & 0.0011 & 0.01794 & 0.00016 & 85.681 & 0.078 & 1.13 & 0.44 & -0.78 & 0.58 & 1.1957 & 0.26 \\
\hline 6750 & 0.11080 & 0.00089 & 0.01765 & 0.00014 & 85.761 & 0.070 & 1.01 & 0.33 & -0.56 & 0.45 & 1.8492 & $2.6 \cdot 10^{-4}$ \\
\hline 7250 & 0.11115 & 0.00072 & 0.01754 & 0.00014 & 85.772 & 0.060 & 0.84 & 0.25 & -0.33 & 0.35 & 2.5861 & $1.27 \cdot 10^{-9}$ \\
\hline 7750 & 0.11109 & 0.00069 & 0.01752 & 0.00013 & 85.776 & 0.058 & 0.85 & 0.25 & -0.38 & 0.35 & 1.3392 & 0.089 \\
\hline 8250 & 0.11076 & 0.00050 & 0.017623 & 0.000080 & 85.778 & 0.040 & 0.99 & 0.20 & -0.61 & 0.27 & 0.99821 & 0.65 \\
\hline 8750 & 0.11116 & 0.00069 & 0.01753 & 0.00012 & 85.761 & 0.056 & 0.85 & 0.27 & -0.46 & 0.37 & 1.3138 & 0.11 \\
\hline 9250 & 0.11083 & 0.00089 & 0.01731 & 0.00018 & 85.839 & 0.079 & 0.63 & 0.28 & -0.16 & 0.40 & 1.1495 & 0.338 \\
\hline 9750 & 0.1120 & 0.0024 & 0.01735 & 0.00061 & 85.78 & 0.21 & 0.31 & 0.44 & 0.26 & 0.73 & 1.5716 & 0.0089 \\
\hline 10250 & 0.1124 & 0.0033 & 0.01736 & 0.00076 & 85.74 & 0.27 & 0.26 & 0.48 & 0.33 & 0.87 & 0.88100 & 0.86 \\
\hline
\end{tabular}

values decrease, on average, with increasing wavelength. However, the observed $x(\lambda)$ are systematically lower than the theoretical values. This is in qualitative agreement with the results obtained for the HD 209458 system [1, 4.

However, the behavior of the observed $x(\lambda)$ differs from the $x(\lambda)$ relation obtained for HD 209458, where the difference between the observed and theoretical $x(\lambda)$ values increases monotonically with $\lambda$; for the HD 189733 system, the difference is largest at the shortest wavelengths and decreases toward longer wavelengts. Figures 4 and 5 indicate that the results obtained when fitting the left and right branches of the HD 189733 light curve separately agree with each other, despite the systematic shift in brightness between these light curves. Therefore, we conclude that our results for the limb-darkening of the star are stable against systematic errors affecting the light curve in the HD 189733 system.

Table 12: Joint fitting of the right and left branches of the observed light curves for HD 189733 [6] with the quadratic limb-darkening law. (Parameter uncertainties estimated using the differential-correction method are given at the $2 \sigma$ level. The two last columns present the values of the reduced $\chi^{2}$ and the corresponding critical significance levels $\alpha_{0}$ ).

\begin{tabular}{|c|c|c|c|c|c|c|c|c|c|c|c|c|}
\hline$\lambda(\stackrel{\circ}{A})$ & $r_{s}^{c}$ & $2 \sigma_{\text {est }}\left(r_{s}^{c}\right)$ & $r_{p}^{c}$ & $2 \sigma_{\text {est }}\left(r_{p}^{c}\right)$ & $i^{c}\left({ }^{\circ}\right)$ & $2 \sigma_{\text {est }}\left(i^{c}\right)\left({ }^{\circ}\right)$ & $x_{1}^{c}$ & $2 \sigma_{\text {est }}\left(x_{1}^{c}\right)$ & $y_{1}^{c}$ & $2 \sigma_{\text {est }}\left(y_{1}^{c}\right)$ & $\chi_{\text {red }}^{2}$ & $\alpha_{0}$ \\
\hline 5750 & 0.11210 & 0.00067 & 0.01760 & 0.00015 & 85.705 & 0.058 & 0.57 & 0.18 & -0.02 & 0.27 & 5.5682 & 0 \\
\hline 6250 & 0.11228 & 0.00094 & 0.01792 & 0.00017 & 85.637 & 0.072 & 0.85 & 0.33 & -0.36 & 0.45 & 0.99780 & 0.608 \\
\hline 6750 & 0.11183 & 0.00086 & 0.01768 & 0.00018 & 85.697 & 0.072 & 0.72 & 0.26 & -0.15 & 0.37 & 1.5358 & 0.00013 \\
\hline 7250 & 0.11156 & 0.00071 & 0.01756 & 0.00015 & 85.737 & 0.061 & 0.66 & 0.20 & -0.10 & 0.29 & 1.9376 & $8 \cdot 10^{-10}$ \\
\hline 7750 & 0.11180 & 0.00072 & 0.01759 & 0.00015 & 85.716 & 0.061 & 0.63 & 0.21 & -0.09 & 0.30 & 2.0992 & $2.5 \cdot 10^{-12}$ \\
\hline 8250 & 0.11201 & 0.00069 & 0.01764 & 0.00015 & 85.712 & 0.058 & 0.61 & 0.20 & -0.10 & 0.29 & 2.2615 & $5 \cdot 10^{-15}$ \\
\hline 8750 & 0.11216 & 0.00077 & 0.01761 & 0.00016 & 85.695 & 0.065 & 0.58 & 0.24 & -0.10 & 0.34 & 2.0344 & $2.7 \cdot 10^{-11}$ \\
\hline 9250 & 0.11174 & 0.00090 & 0.01743 & 0.00022 & 85.766 & 0.084 & 0.43 & 0.23 & 0.09 & 0.34 & 1.4995 & $3 \cdot 10^{-4}$ \\
\hline 9750 & 0.11105 & 0.00091 & 0.01736 & 0.00021 & 85.804 & 0.083 & 0.47 & 0.25 & 0.01 & 0.37 & 1.3637 & 0.0063 \\
\hline 10250 & 0.1129 & 0.0023 & 0.01733 & 0.00073 & 85.74 & 0.18 & 0.00 & 0.35 & 0.72 & 0.38 & 0.77561 & 0.98971 \\
\hline
\end{tabular}

HD 189733 and HD 209458 have qualitatively different functions $x(\lambda)$, probably due to the fact that there were spots on the surface of the star in the HD 189733 system at the observing epoch [5, 6]. Therefore, additional observations of HD 189733 in periods of lower activity would be of great interest. Note that the observed $x(\lambda)$ values in both HD 189733 and HD 209458 lie below the theoretical relations, although the observed $x(\lambda)$ relations are qualitatively different for them. This result is important for checking modern models of thin stellar atmospheres. 


\section{FITTING OF THE LIGHT CURVES WITH QUADRATIC LIMB DARK- ENING}

The results of fitting the light curves using the quadratic limb-darkening law are given in Tables 10-16. The data obtained using the differential-correction method are given in Tables 10-12. Here, the central parameter values $r_{p}, r_{s}, i, x_{1}, y_{1}$ together with their $2 \sigma$ uncertainties $(\gamma=95.5 \%)$ are shown. Tables 10-12 also contain the values of $\chi_{\text {red }}^{2}$ and $\alpha_{0}$. Fitting of the left and right branches of the light curves (Tables 10 and 11) yields $\chi_{\text {red }}^{2}<1$ and $\alpha_{0}>0.5$ for some wavelengths. This indicates that the observed brightnesses may be correlated and systematic errors may be present in the observational data. When we fit the light curve as a whole (Table 12), our model is "bad" for most wavelengths, and is rejected at a very low significance level. The light-curve fits at $\lambda=6250 \AA$ and $\lambda=10250 \AA$ yield $\chi_{\text {red }}^{2} \leq 1$ and $\alpha_{0}>0.5$, suggesting a strong correlation of the observational points. All these factors lead us to adopt $2 \sigma_{\text {est }}$ rather than $\sigma_{\text {est }}$ to estimate the uncertainties of the model parameters for the quadratic limb-darkening law, as was done for the linear limb-darkening model.

Table 13: Fitting of the left branches of the observed light curves for HD 189733 [6] with the quadratic limb-darkening law. (Parameter uncertainties were estimated using the confidence-area method based on the $\chi_{P}^{2}$ distribution, with $\gamma=0.955$.)

\begin{tabular}{c|c|c|c|c|c|c|c|c|c|c}
$\lambda(\AA)$ & $r_{s}$ & $\Delta_{P}\left(r_{s}\right)$ & $r_{p}$ & $\Delta_{P}\left(r_{p}\right)$ & $i$ & $\Delta_{P}(i)$ & $x_{1}$ & $\Delta_{P}\left(x_{1}\right)$ & $y_{1}$ & $\Delta_{P}\left(y_{1}\right)$ \\
\hline 5750 & 0.11212 & 0.00067 & 0.01754 & 0.00016 & $85.722^{\circ}$ & $0.061^{\circ}$ & 0.43 & 0.18 & 0.17 & 0.27 \\
6750 & 0.1123 & 0.0020 & 0.01757 & 0.00047 & $85.71^{\circ}$ & $0.18^{\circ}$ & 0.51 & 0.48 & 0.13 & 0.73 \\
7250 & 0.1116 & 0.0013 & 0.01747 & 0.00031 & $85.76^{\circ}$ & $0.12^{\circ}$ & 0.51 & 0.32 & 0.07 & 0.49 \\
7750 & 0.1120 & 0.0013 & 0.01752 & 0.00028 & $85.72^{\circ}$ & $0.11^{\circ}$ & 0.45 & 0.32 & 0.08 & 0.48 \\
8250 & 0.1126 & 0.0012 & 0.01754 & 0.00029 & $85.72^{\circ}$ & $0.12^{\circ}$ & 0.32 & 0.34 & 0.29 & 0.51 \\
8750 & 0.1125 & 0.0014 & 0.01758 & 0.00030 & $85.69^{\circ}$ & $0.12^{\circ}$ & 0.41 & 0.37 & 0.09 & 0.54 \\
9250 & 0.1122 & 0.0015 & 0.01745 & 0.00038 & $85.76^{\circ}$ & $0.15^{\circ}$ & 0.32 & 0.45 & 0.20 & 0.67 \\
9750 & 0.1112 & 0.0018 & 0.01736 & 0.00039 & $85.80^{\circ}$ & $0.16^{\circ}$ & 0.43 & 0.39 & 0.13 & 0.62 \\
\hline
\end{tabular}

Table 14: Fitting of the left branches of the observed light curves for HD 189733 [6] with the quadratic limb-darkening law. (Parameter uncertainties were estimated using the confidence-area method based on the $\chi_{M}^{2}$ distribution, with $\left.\gamma=0.955\right)$.

\begin{tabular}{c|c|c|c|c|c|c|c|c|c|c}
$\lambda(\AA)$ & $r_{s}$ & $\Delta_{M}\left(r_{s}\right)$ & $r_{p}$ & $\Delta_{M}\left(r_{p}\right)$ & $i$ & $\Delta_{M}(i)$ & $x_{1}$ & $\Delta_{M}\left(x_{1}\right)$ & $y_{1}$ & $\Delta_{M}\left(y_{1}\right)$ \\
\hline 6750 & 0.1150 & 0.0066 & 0.01785 & 0.00051 & $85.77^{\circ}$ & $0.41^{\circ}$ & 1.27 & 0.81 & 1.3 & 1.3 \\
7250 & 0.1115 & 0.0022 & 0.01758 & 0.00039 & $85.77^{\circ}$ & $0.21^{\circ}$ & 0.63 & 0.40 & 0.23 & 0.62 \\
7750 & 0.1118 & 0.0014 & 0.01756 & 0.00030 & $85.71^{\circ}$ & $0.12^{\circ}$ & 0.47 & 0.27 & 0.13 & 0.42 \\
8750 & 0.1123 & 0.0018 & 0.01766 & 0.00036 & $85.69^{\circ}$ & $0.17^{\circ}$ & 0.51 & 0.36 & 0.28 & 0.55 \\
9250 & 0.1121 & 0.0022 & 0.01762 & 0.00036 & $85.76^{\circ}$ & $0.22^{\circ}$ & 0.42 & 0.35 & 0.36 & 0.56 \\
9750 & 0.1109 & 0.0018 & 0.01741 & 0.00040 & $85.77^{\circ}$ & $0.14^{\circ}$ & 0.39 & 0.40 & 0.073 & 0.64 \\
\hline
\end{tabular}

Tables 13, 15, 16, show the results of fitting the light curves of HD 189733 using the model with quadratic limb darkening; the parameter uncertainties are estimated using the confidence-area method based on the $\chi_{P}^{2}$ distribution. Table 14 presents the results of 
Table 15: Fitting of the right branches of the observed light curves for HD 189733 [6] with the quadratic limb-darkening law. (Parameter uncertainties were estimated using the confidence-area method based on the $\chi_{P}^{2}$ distribution, with $\gamma=0.955$.)

\begin{tabular}{c|c|c|c|c|c|c|c|c|c|c}
$\lambda(\AA)$ & $r_{s}$ & $\Delta_{P}\left(r_{s}\right)$ & $r_{p}$ & $\Delta_{P}\left(r_{p}\right)$ & $i$ & $\Delta_{P}(i)$ & $x_{1}$ & $\Delta_{P}\left(x_{1}\right)$ & $y_{1}$ & $\Delta_{P}\left(y_{1}\right)$ \\
\hline 5750 & 0.11091 & 0.00070 & 0.01752 & 0.00016 & $85.782^{\circ}$ & $0.060^{\circ}$ & 0.87 & 0.18 & -0.44 & 0.26 \\
6250 & 0.1109 & 0.0028 & 0.01784 & 0.00064 & $85.73^{\circ}$ & $0.22^{\circ}$ & 1.27 & 0.69 & -0.73 & 0.97 \\
6750 & 0.1108 & 0.0019 & 0.01758 & 0.00043 & $85.77^{\circ}$ & $0.16^{\circ}$ & 1.02 & 0.44 & -0.60 & 0.64 \\
7250 & 0.1111 & 0.0014 & 0.01750 & 0.00037 & $85.78^{\circ}$ & $0.13^{\circ}$ & 0.85 & 0.37 & -0.39 & 0.54 \\
7750 & 0.1111 & 0.0013 & 0.01751 & 0.00030 & $85.78^{\circ}$ & $0.11^{\circ}$ & 0.87 & 0.33 & -0.38 & 0.48 \\
8250 & 0.1108 & 0.0012 & 0.01763 & 0.00027 & $85.778^{\circ}$ & $0.098^{\circ}$ & 1.01 & 0.29 & -0.59 & 0.42 \\
8750 & 0.1113 & 0.0015 & 0.01753 & 0.00032 & $85.76^{\circ}$ & $0.12^{\circ}$ & 0.85 & 0.38 & -0.45 & 0.55 \\
9250 & 0.1109 & 0.0016 & 0.01731 & 0.00041 & $85.84^{\circ}$ & $0.16^{\circ}$ & 0.66 & 0.45 & -0.12 & 0.67 \\
9750 & 0.1108 & 0.0020 & 0.01726 & 0.00043 & $85.85^{\circ}$ & $0.18^{\circ}$ & 0.55 & 0.53 & -0.17 & 0.78 \\
\hline
\end{tabular}

Table 16: Joint fitting of the right and left branches of the observed light curves for HD189733 [6] with the quadratic limbdarkening law. (Parameter uncertainties were estimated using the confidence-area method based on the $\chi_{P}^{2}$ distribution, with $\gamma=0.955$.)

\begin{tabular}{c|c|c|c|c|c|c|c|c|c|c}
$\lambda(\AA)$ & $r_{s}$ & $\Delta_{P}\left(r_{s}\right)$ & $r_{p}$ & $\Delta_{P}\left(r_{p}\right)$ & $i$ & $\Delta_{P}(i)$ & $x_{1}$ & $\Delta_{P}\left(x_{1}\right)$ & $y_{1}$ & $\Delta_{P}\left(y_{1}\right)$ \\
\hline 5750 & 0.11182 & 0.00048 & 0.01757 & 0.00011 & $85.724^{\circ}$ & $0.042^{\circ}$ & 0.60 & 0.12 & -0.09 & 0.18 \\
6250 & 0.1124 & 0.0021 & 0.01790 & 0.00047 & $85.64^{\circ}$ & $0.17^{\circ}$ & 0.88 & 0.53 & -0.35 & 0.76 \\
6750 & 0.1119 & 0.0014 & 0.01765 & 0.00031 & $85.71^{\circ}$ & $0.12^{\circ}$ & 0.71 & 0.34 & -0.16 & 0.50 \\
7250 & 0.11160 & 0.00095 & 0.01755 & 0.00023 & $85.741^{\circ}$ & $0.085^{\circ}$ & 0.65 & 0.24 & -0.12 & 0.36 \\
7750 & 0.11183 & 0.00091 & 0.01758 & 0.00019 & $85.718^{\circ}$ & $0.075^{\circ}$ & 0.62 & 0.23 & -0.10 & 0.34 \\
8250 & 0.11204 & 0.00085 & 0.01763 & 0.00019 & $85.714^{\circ}$ & $0.072^{\circ}$ & 0.61 & 0.22 & -0.11 & 0.32 \\
8750 & 0.11219 & 0.00100 & 0.01760 & 0.00021 & $85.697^{\circ}$ & $0.082^{\circ}$ & 0.58 & 0.27 & -0.10 & 0.38 \\
9250 & 0.1118 & 0.0011 & 0.01743 & 0.00028 & $85.77^{\circ}$ & 0.11 & 0.45 & 0.33 & 0.12 & 0.48 \\
9750 & 0.1111 & 0.0013 & 0.01735 & 0.00027 & $85.81^{\circ}$ & 0.11 & 0.48 & 0.35 & 0.01 & 0.51 \\
\hline
\end{tabular}

fitting the left branches of the light curves using the quadratic limb-darkening law. Here, the parameter uncertainties were obtained using the confidence-area method based on the $\chi_{M}^{2}$ distribution $(\gamma=0.955)$.

\section{$x_{1}$ AND $y_{1}$ AS FUNCTIONS OF WAVELENGTH IN THE QUADRATIC LIMB-DARKENING LAW}

Figures 8-13 show the observed $x_{1}(\lambda)$ and $y_{1}(\lambda)$ values as functions of wavelength, obtained using the differential-correction method. The $2 \sigma$ uncertainties are given $(\gamma=$ 95.5\%). The theoretical relations $x_{1}(\lambda)$ and $y_{1}(\lambda)$ obtained in [2, 15] are also shown here. The observed relation $x_{1}(\lambda)$ obtained using the left branch of the light curve agrees with the theoretical function $x_{1}(\lambda)$, while the observed $x_{1}(\lambda)$ obtained using the right branch of the light curve lies considerably above the theoretical relation (Figs, 8 and 9). The observed $x_{1}(\lambda)$ obtained using the whole light curve agrees satisfactorily with the theoretical relation within the uncertainties (at the $2 \sigma$ level).

The observed $y_{1}(\lambda)$ obtained using the left branch of the light curve agrees with the 
theoretical function, while the observed $y_{1}(\lambda)$ obtained for the right branch of the light curve lies considerably below the theoretical relation. The observed $y_{1}(\lambda)$ obtained using the whole light curve agrees satisfactorily with the theoretical relation within the uncertainties (at the $2 \sigma$ level) (Figs. 11- 13).

Note that the observed relations $x_{1}(\lambda)$ and $y_{1}(\lambda)$ obtained using the whole light curve do not agree with the theoretical relations at the $1 \sigma$ level: the observed $x_{1}(\lambda)$ is systematically higher than the theoretical relation, and $y_{1}(\lambda)$ is lower than the theoretical relation (Figs. 10 and 13). Since our model is formally bad and, moreover, has five rather than one parameters, we are forced to take the uncertainties at the $2 \sigma$ level. With these uncertainties for the coefficients $x_{1}(\lambda)$ and $y_{1}(\lambda)$, the differences between the observed and theoretical $x_{1}(\lambda)$ and $y_{1}(\lambda)$ relations do not seem to be significant. Figures 14 and 15 show the $x_{1}(\lambda)$ and $y_{1}(\lambda)$ relations obtained when fitting the left and right branches of the light curve, with the uncertainties derived using the confidence-area method based on the $\chi_{P}^{2}$ distribution.

Figures 16 and 17 illustrate the projections of the confidence areas in the $\left(r_{s}, r_{p}\right)$ and $\left(x_{1}, y_{1}\right)$ planes obtained when using the $\chi_{P}^{2}$ and $\chi_{M}^{2}$ distributions. The light curve at $\lambda 9500 \div 10000 \AA$ (left branch) was used with the model based on the quadratic limbdarkening law.

\section{CONCLUSION}

We have analyzed high-accuracy multicolor light curves given in [5, 6] for the starexplanet system HD 189733 and determined the radii of the star and planet, the orbital inclination, and the coefficients in the linear and quadratic limb-darkening laws across the disk of the K2V star. The results of our fitting agree with the data obtained in [5, 6].

We have analyzed in detail the limb darkening across the disk of the K2V star based on the resulting uncertainties in the coefficients in the linear and quadratic limb-darkening laws. We allowed for the presence of spots on the surface of the K2V star [6] by analyzing the left and right branches of the light curves separately, as well as the light curve as a whole. Moreover, we paid special attention to checking the adequacy of our model and verifying how well it agrees with the observational data. Our model proved to be formally "bad". When fitting the left and right branches of the light curve separately, our model is rejected at a high significance level of $\alpha_{0}>50 \%$ at some wavelengths; this most likely indicates a correlation of the individual observational points in the light curve. When fitting the light curve as a whole, our model is rejected at a very low significance level for most wavelengths.

Since our model turned out to be "bad" when applied to HD 189733, we were forced to adopt the high confidence level $\gamma=95.5 \%$ (rather than the $68 \%$ relevant for "good" models) when estimating the parameter uncertainties. Since our model includes four or five parameters instead of only one, we choose the error intervals in the confidence-area method such that the probability for them to encompass the true parameter values is undoubtedly higher than the given probability (in this case, the given confidence level $\gamma=95.5 \%$ is associated with the entire confidence area $D$ rather than only one confidence interval). Our analysis of the relations $x(\lambda), x_{1}(\lambda), y_{1}(\lambda)$ for the limb-darkening coefficients deduced from fitting the observations yielded the following results $\gamma=95.5 \%$. 
The observed values of the coefficient $x(\lambda)$ in the linear limb-darkening law for HD 189733 are systematically below the theoretical relation, with the differences increasing with decreasing wavelength $\lambda$ (in contrast to HD 209458, where they increase with increasing $\lambda$ ). The observational coefficients in the quadratic limb-darkening law $x_{1}(\lambda)$ and $y_{1}(\lambda)$ agree satisfactorily within the $2 \sigma$ uncertainties $(\gamma=95.5 \%)$ with the theoretical relations developed for one-dimensional thin stellar atmospheres ([2, 3, 15]).

We emphasize that these conclusions concern the light curves of the HD 189733 system when there were spots on the disk of the K2V star. To further invetigate limb darkening on this star, additional observational data should be obtained for eclipse curves at epochs when the contribution of spots is negligible.

We confirm the earlier conclusion [5] (at the $1 \sigma$ level) that the exoplanet radius increases with decreasing wavelength. This may imply the presence of the atmosphere around the exoplanet.

The authors are grateful to Frederic Pont for providing us with the observational data on HD 189733. 


\section{References}

[1] M.K. Abubekerov, N.Yu. Gostev, and A. M. Cherepashchuk, Astron. Rep. 54, 1105 (2010).

[2] A. Claret, Astron \& Astrophys 428, 1001 (2004).

[3] A. Claret, Astron \& Astrophys 506, 1335 (2009).

[4] J. Southworth, Monthly Not. Roy. Astron. Soc. 386, 1644 (2008).

[5] F.Pont, R.L. Gilliland, C. Moutou et al., Astron \& Astrophys 476, 1347 (2007).

[6] F. Pont, H. Knutson, R. L. Gilliland et al., Monthly Not. Roy. Astron. Soc. 385, 109 (2008).

[7] F. Bouchy, S. Udry, M. Mayor et al., Astron \& Astrophys 444, 15 (2005).

[8] I. Kudzei, Astron. Tsirk., No. 1363 (1985).

[9] S. Kasuya, M.Honda, R. Mishima, Monthly Not. Roy. Astron. Soc. 411, 1863 (2011).

[10] M.K. Abubekerov, N.Yu. Gostev, and A.M. Cherepashchuk, Astron. Rep. 52, 99 (2008).

[11] M.K. Abubekerov, N.Yu. Gostev, and A.M. Cherepashchuk, Astron. Rep. 53, 722 (2009).

[12] D.M. Popper, Astron.J. 89, 132 (1984).

[13] A.M. Cherepashchuk, Astron. Rep. 37, 585 (1993).

[14] A. Claret, Astron \& Astrophys 335, 647 (1998).

[15] A. Claret, Astron \& Astrophys 363, 1081 (2000).

[16] Eggleton P.P., Astrophys.J. 268, 368 (1983). 


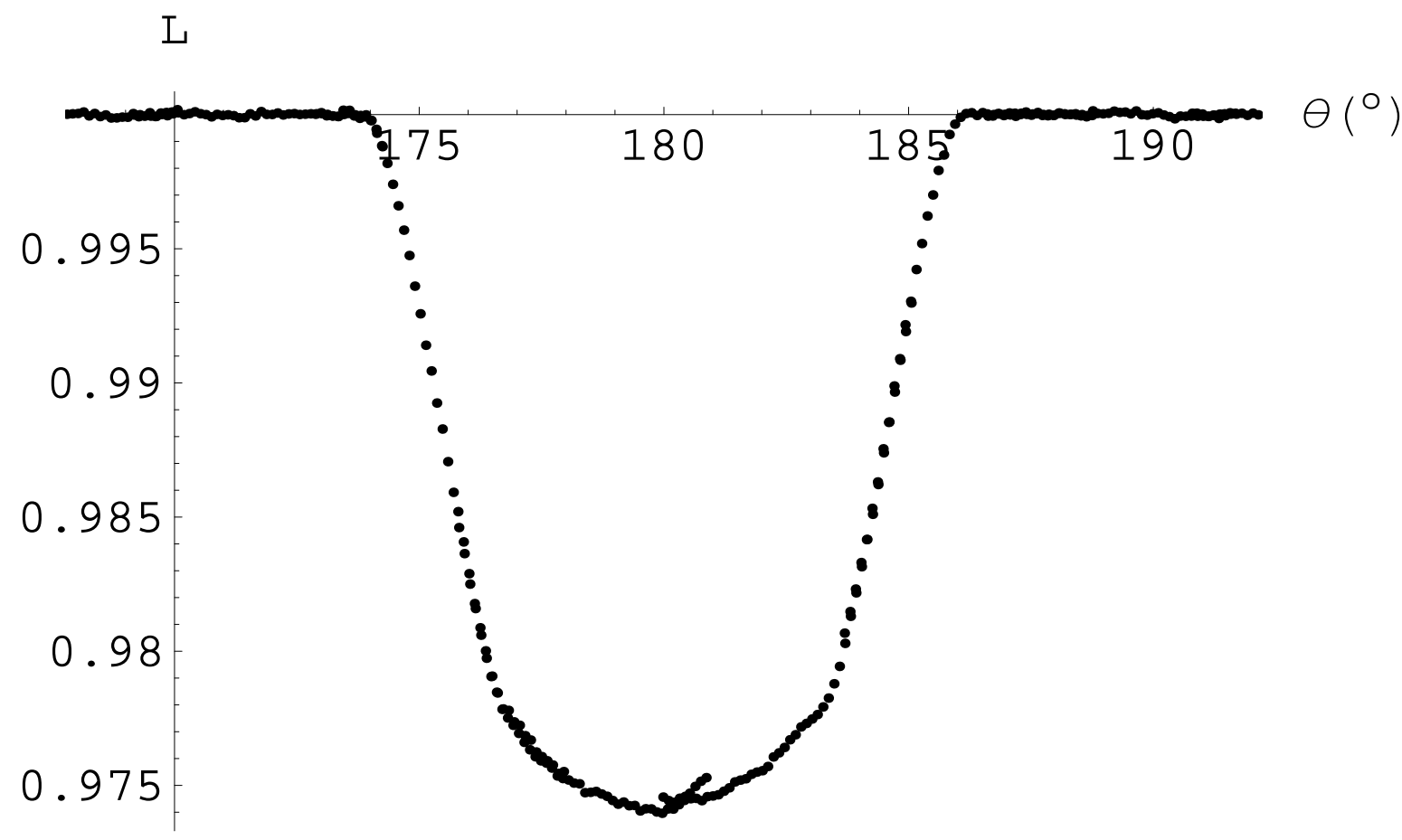

Fig 1: Light curve 1 of the star HD 189733 in the filter $\lambda=5500-6000$ taken from [6]. The light curve has truncated wings and a brightness jump at the minimum (due to a spot on the surface of the star). 


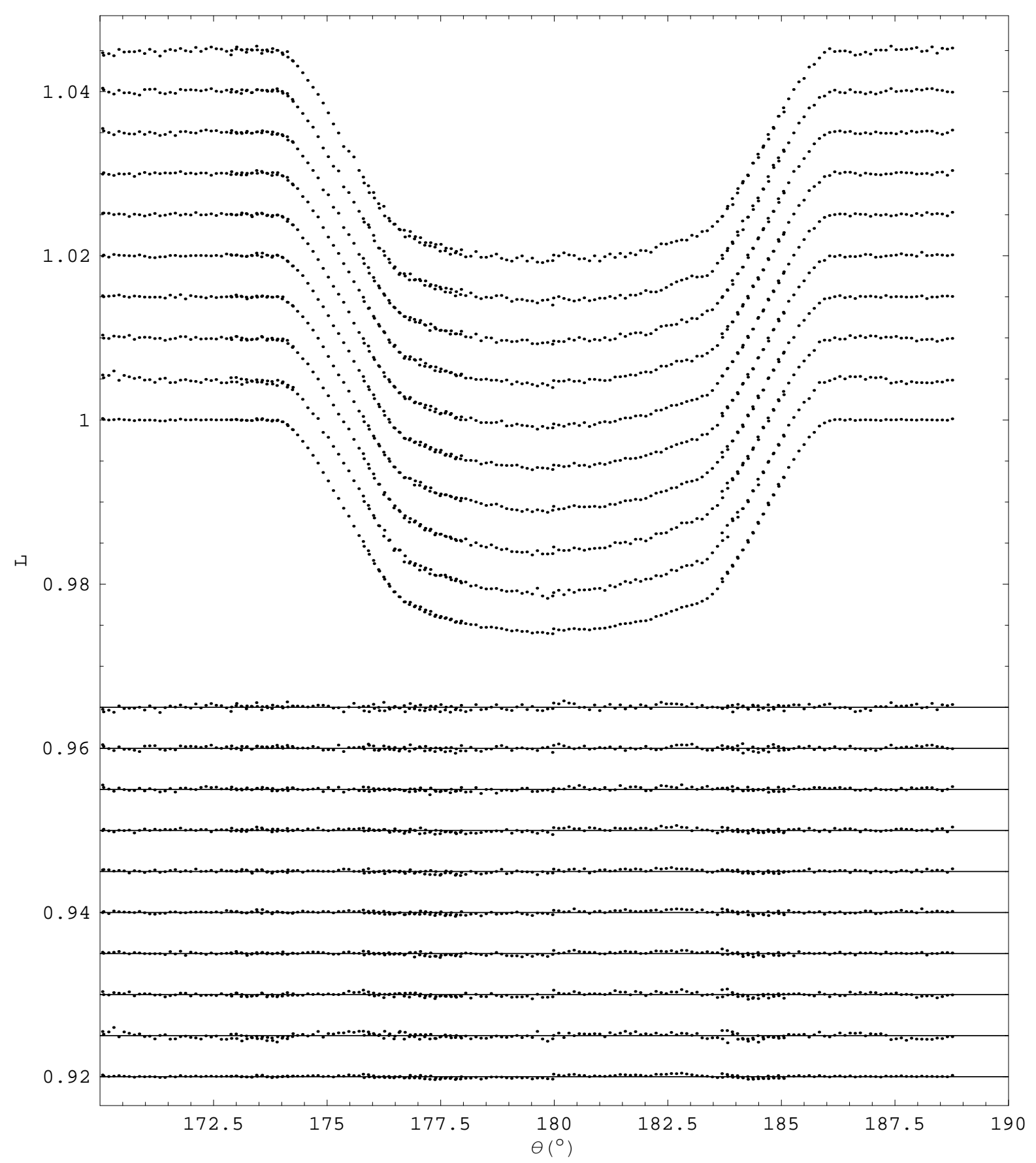

Fig 2: Light curves of HD 189733 at $\lambda=5500-10500$. The wavelenght increases upward. The residuals for the quadratic limb-darkening law with the best-fit parameter values are given in the lower part. 


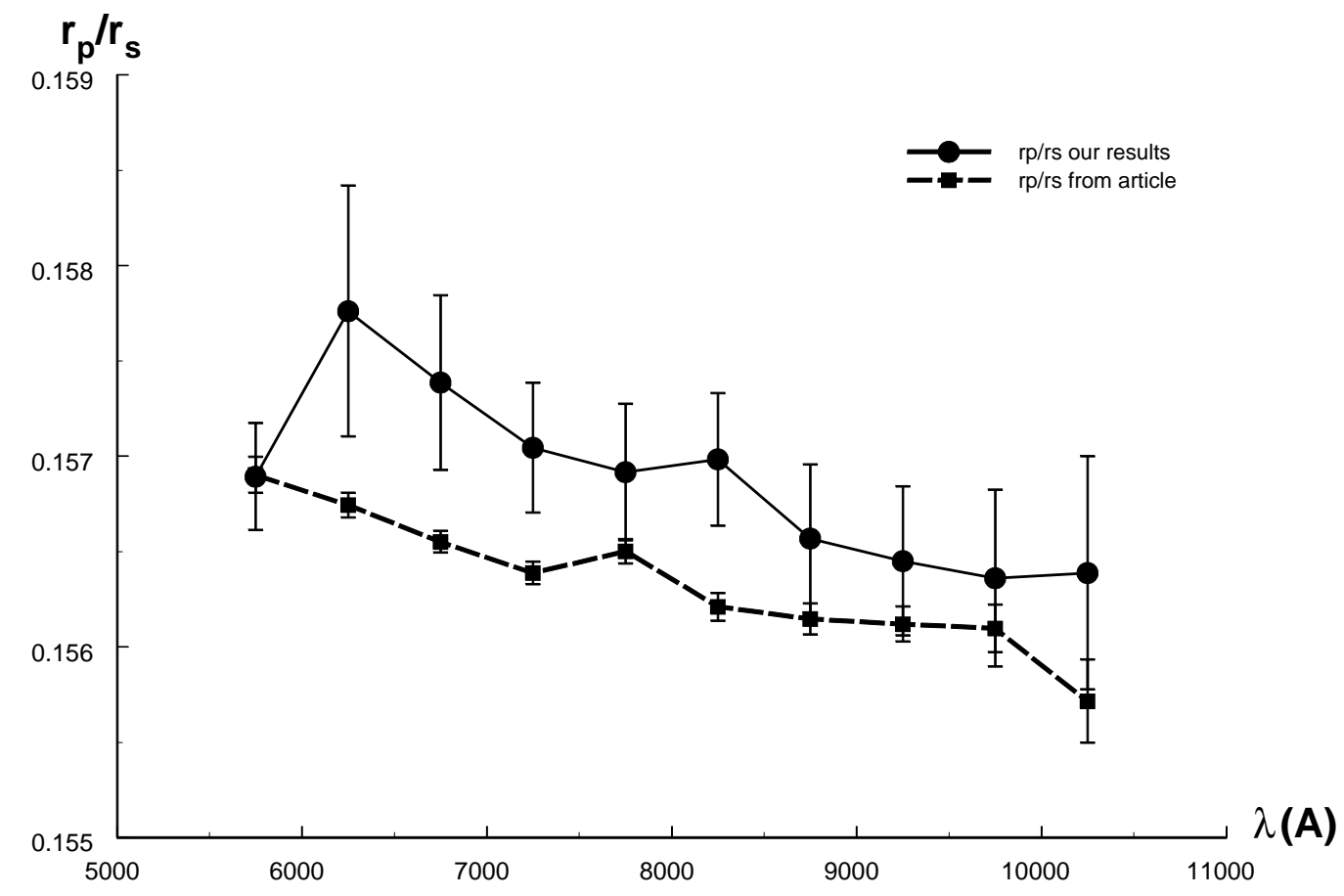

Fig 3: The planet-to-star radius ratio as a function of wavelength obtained in this paper (filled circles) and according to [6] (filled squares). The indicated uncertainties are $1 \sigma$. Since we do not specify the limb-darkening coefficient, and are searching for it together with the other parameters of the problem, our uncertainties are larger. 


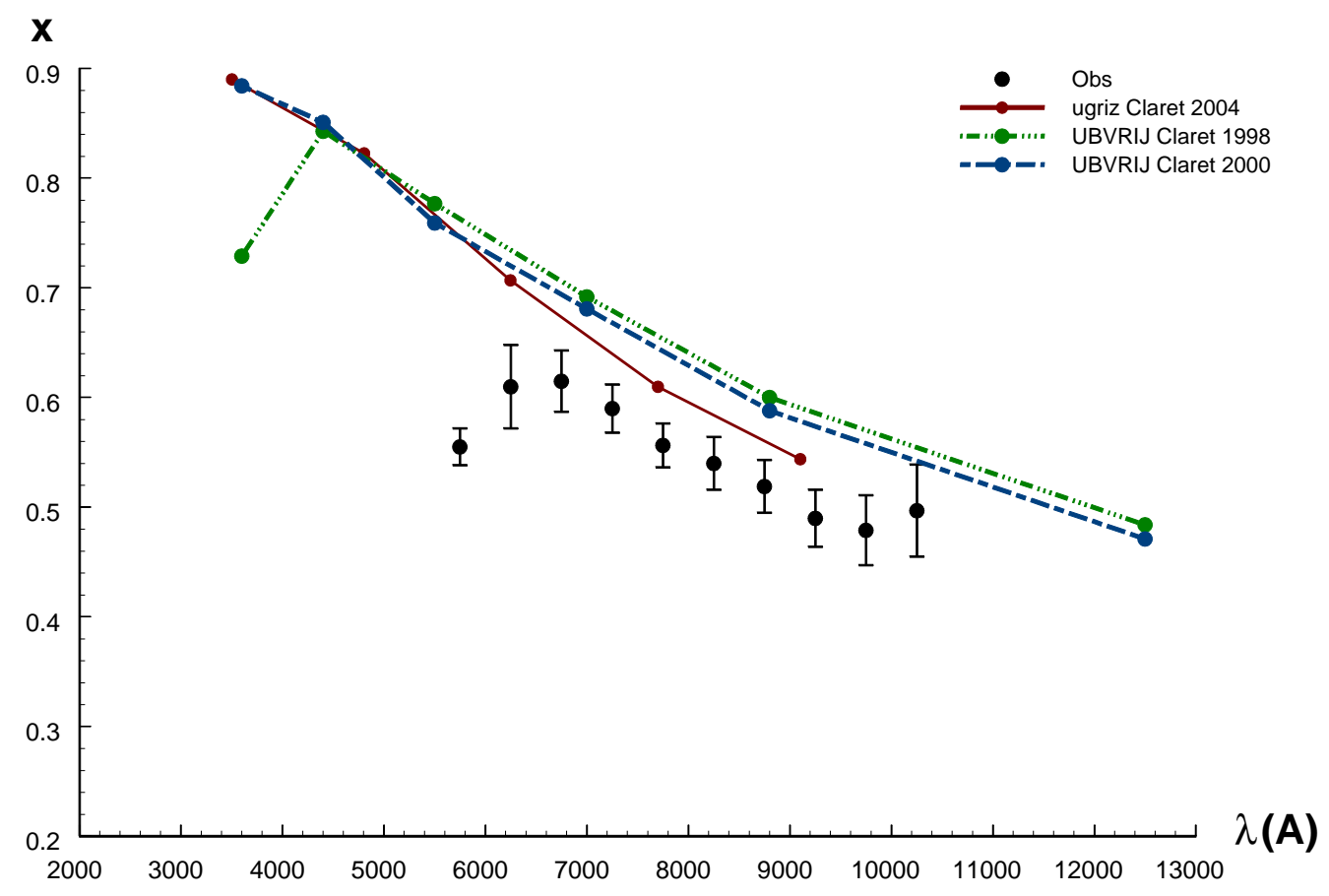

Fig 4: Limb-darkening coefficient $x$ for HD 189733 as a function of wavelength $\lambda$ derived for the linear limb-darkening law and the left branches of the light curves from [6]. The uncertainties of the limb-darkening coefficient were obtained using the differentialcorrection method and are given at the $2 \sigma$ level. The theoretical values of the limbdarkening coefficients in the ugriz and UBVRIJ photometric systems were taken from Claret2004,Claret1998, Claret2000. 


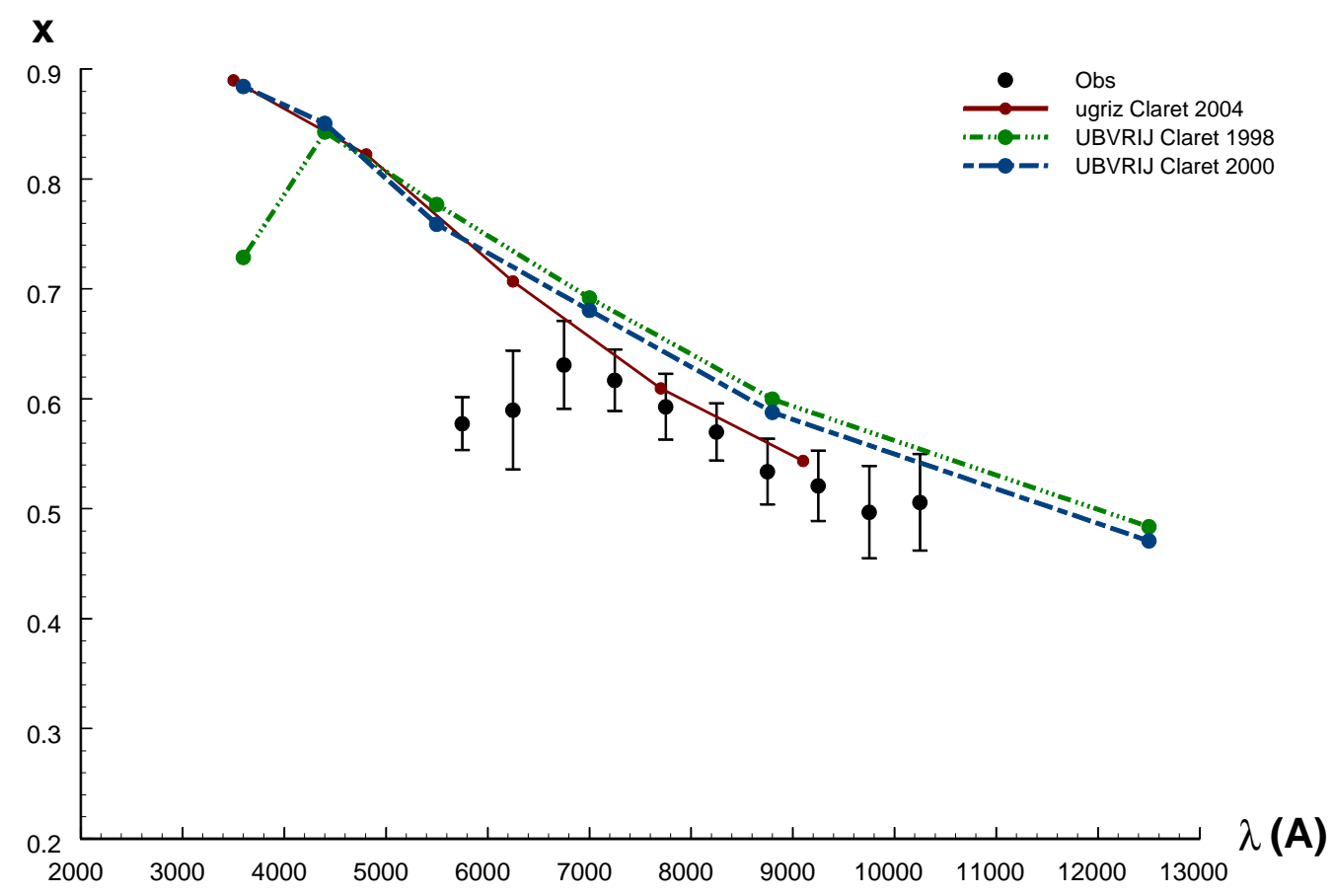

Fig 5: Limb-darkening coefficient $x$ for HD 189733 as a function of wavelength $\lambda$ derived for the linear limb-darkening law and the right branches of the light curves from [6]. The uncertainties of limb-darkening coefficient were obtained using the differential-correction method and are given at the $2 \sigma$ level. The theoretical values of the limb-darkening coefficient in the ugriz and UBV RIJ photometric systems were taken from [2, 14, 15]. 


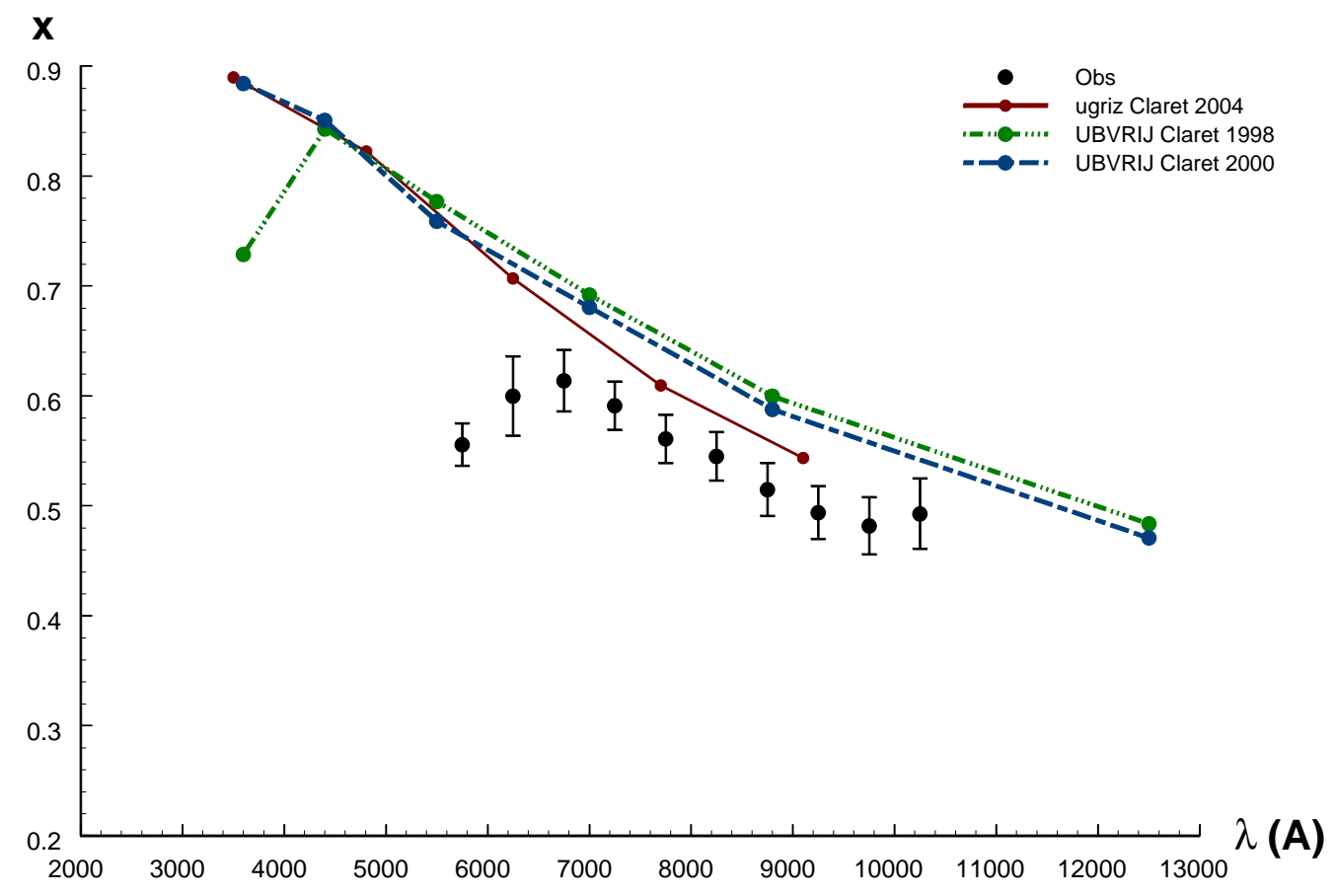

Fig 6: Limb-darkening coefficient $x$ forHD189733 as a function of wavelength $\lambda$ derived for the linear limb-darkening law and the joint analysis of the right and left branches of the light curves from [6]. The uncertainties of limb-darkening coefficient were obtained using the differential-correction method and are given at the 2 level. The theoretical values of the limb-darkening coefficient in the ugriz and UBVRIJ photometric systems were taken from [2, 14, 15]. 


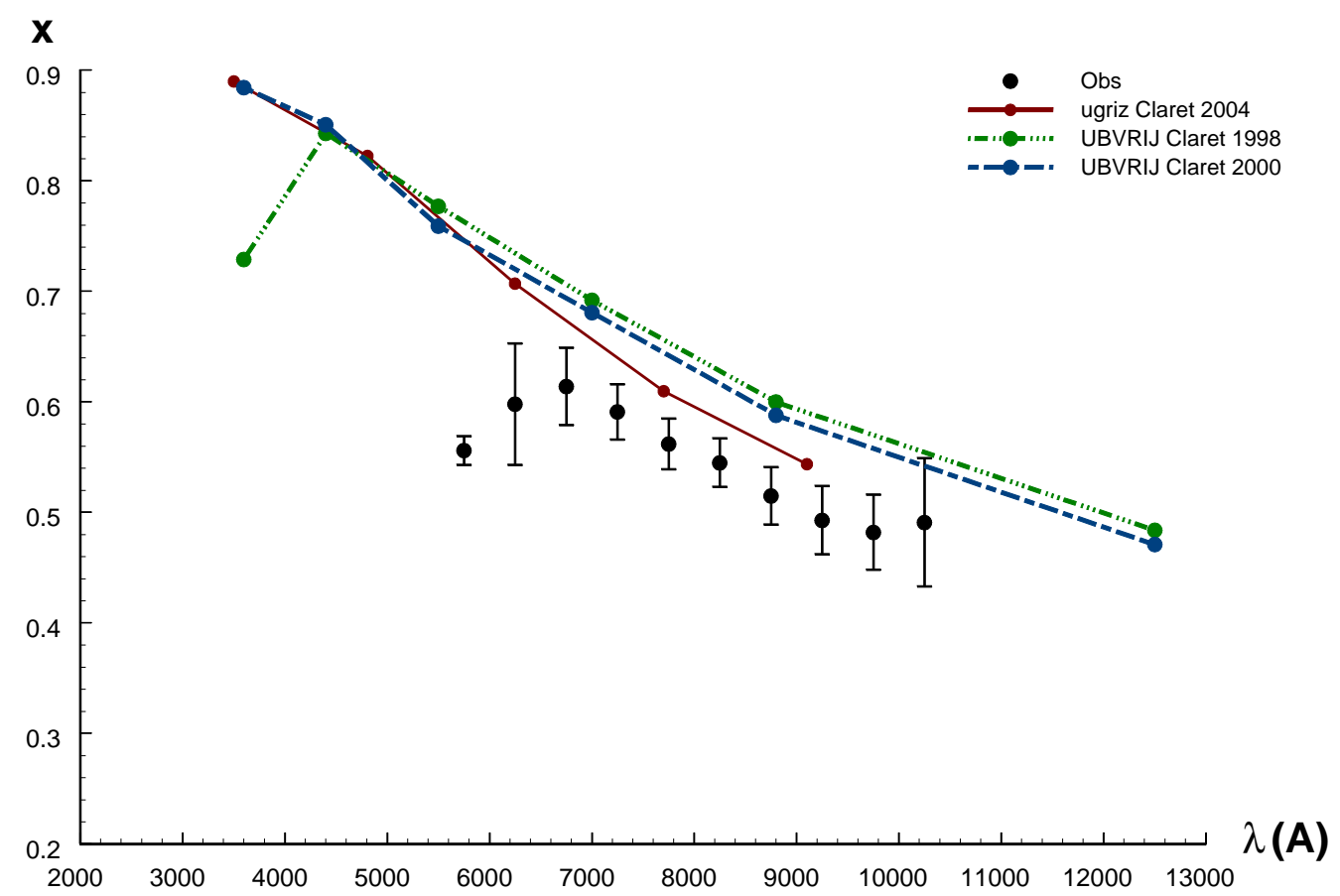

Fig 7: Same function as in Fig.6 but with the uncertainties of the limb-darkening coefficients obtained using the confidencearea method based on the $\chi_{P}^{2}$ distribution; the uncertainties are given for $\gamma=0.955$. 


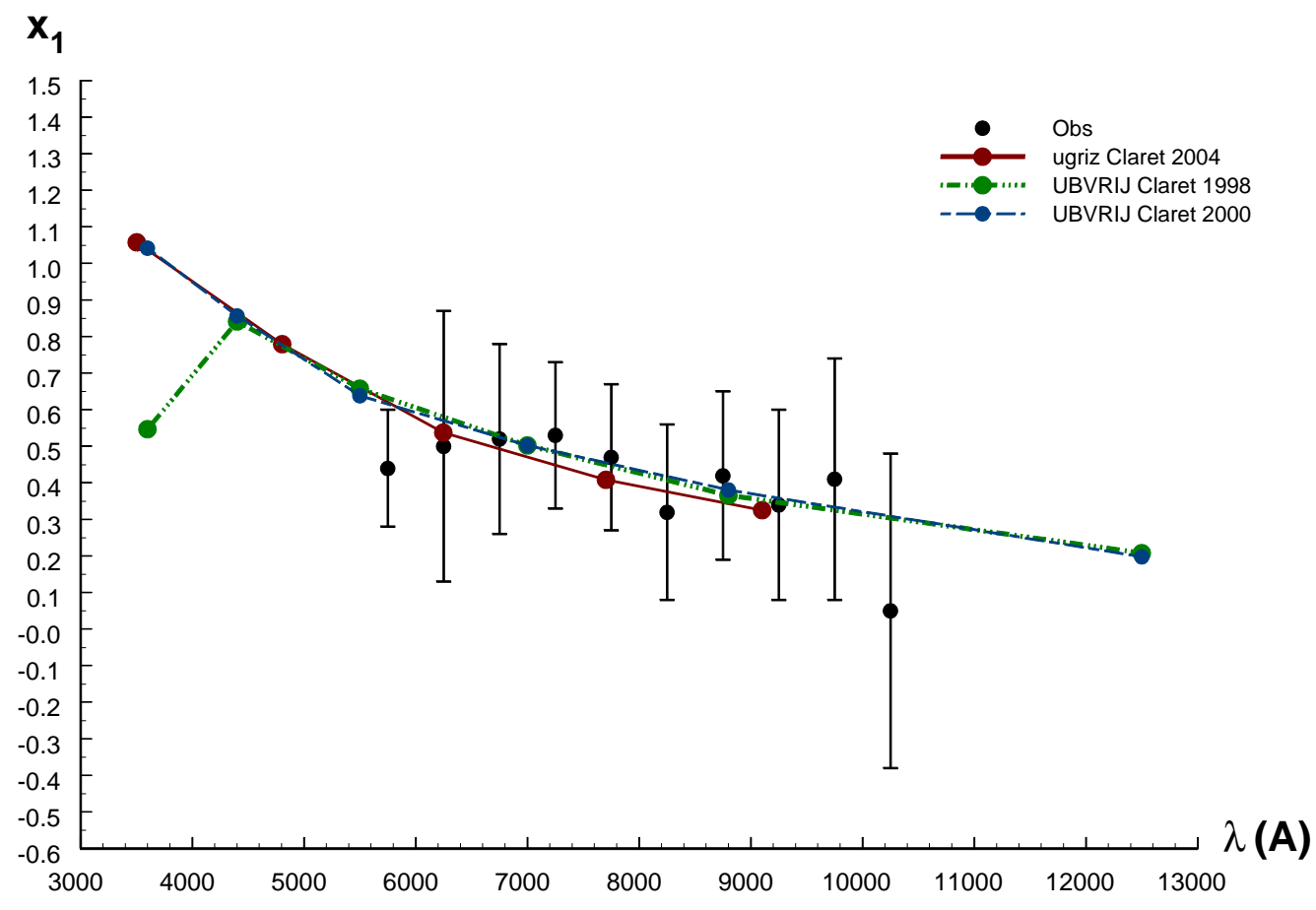

Fig 8: Same function as in Fig.4 but for the linear coefficient $x_{1}$ and the quadratic limbdarkening law. 


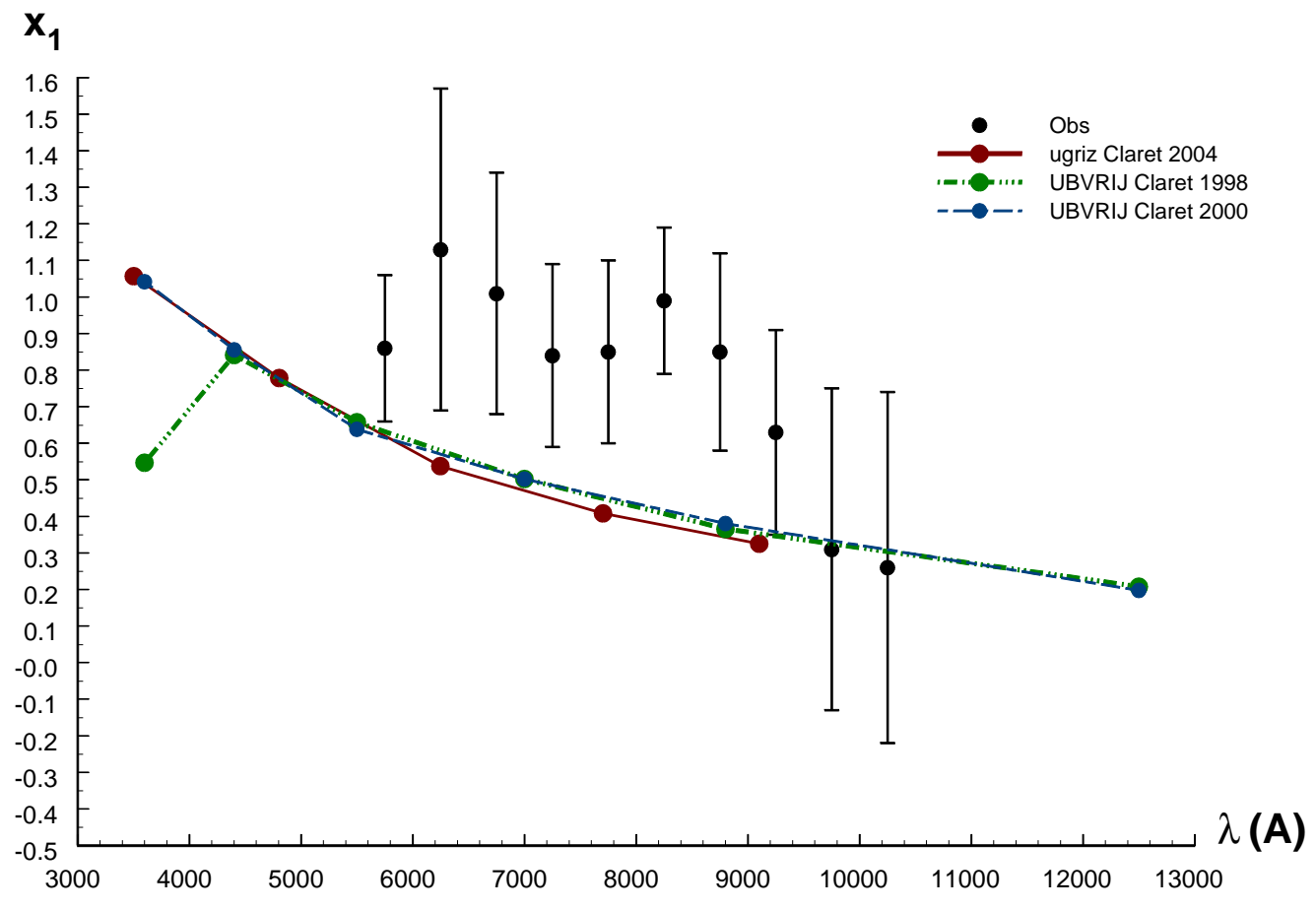

Fig 9: Same function as in Fig. 8 but obtained from the analysis of the right branches of the light curves. 


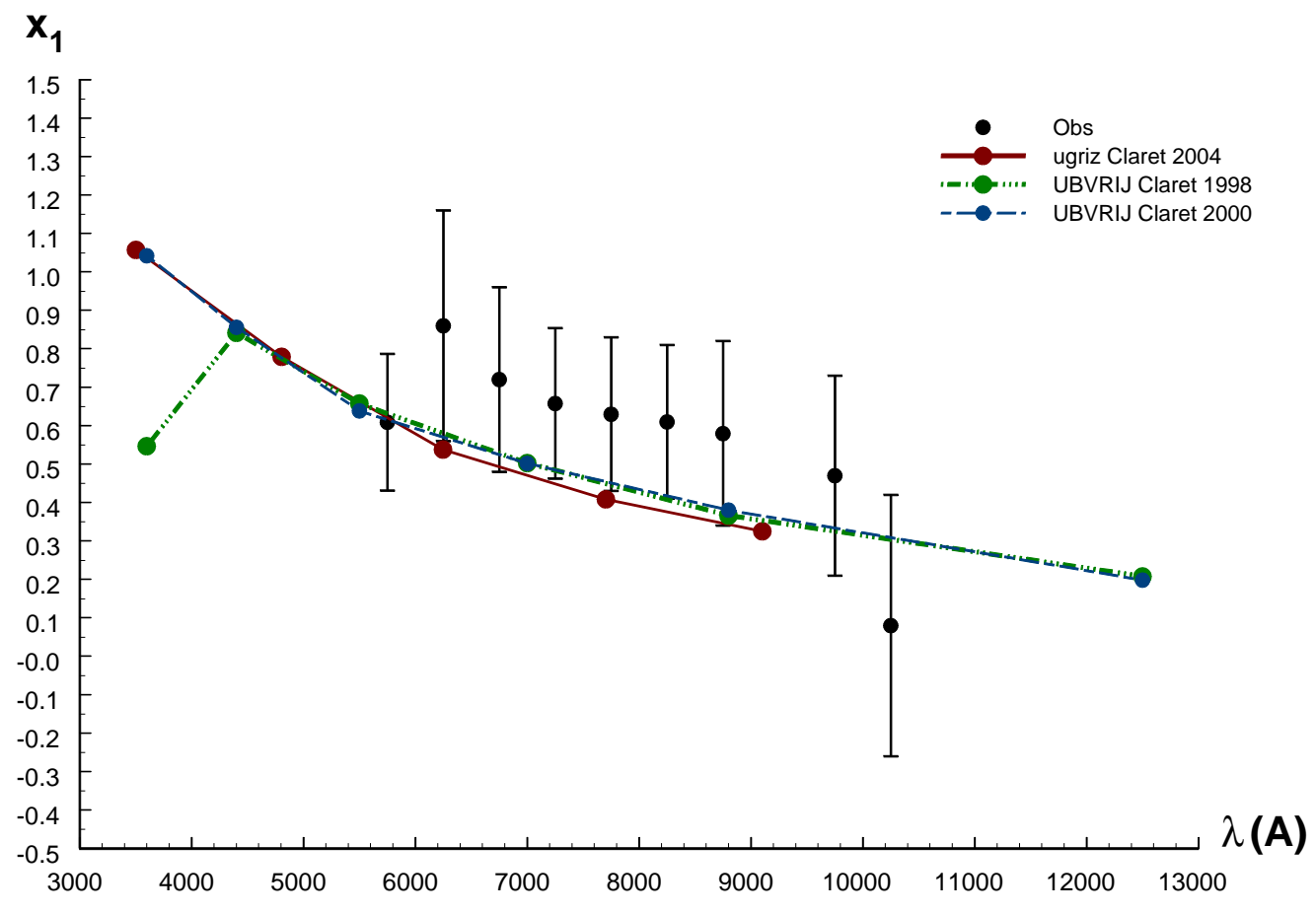

Fig 10: Same function as in Fig. 8 but obtained from the joint analysis of the left and right branches of the light curves. 


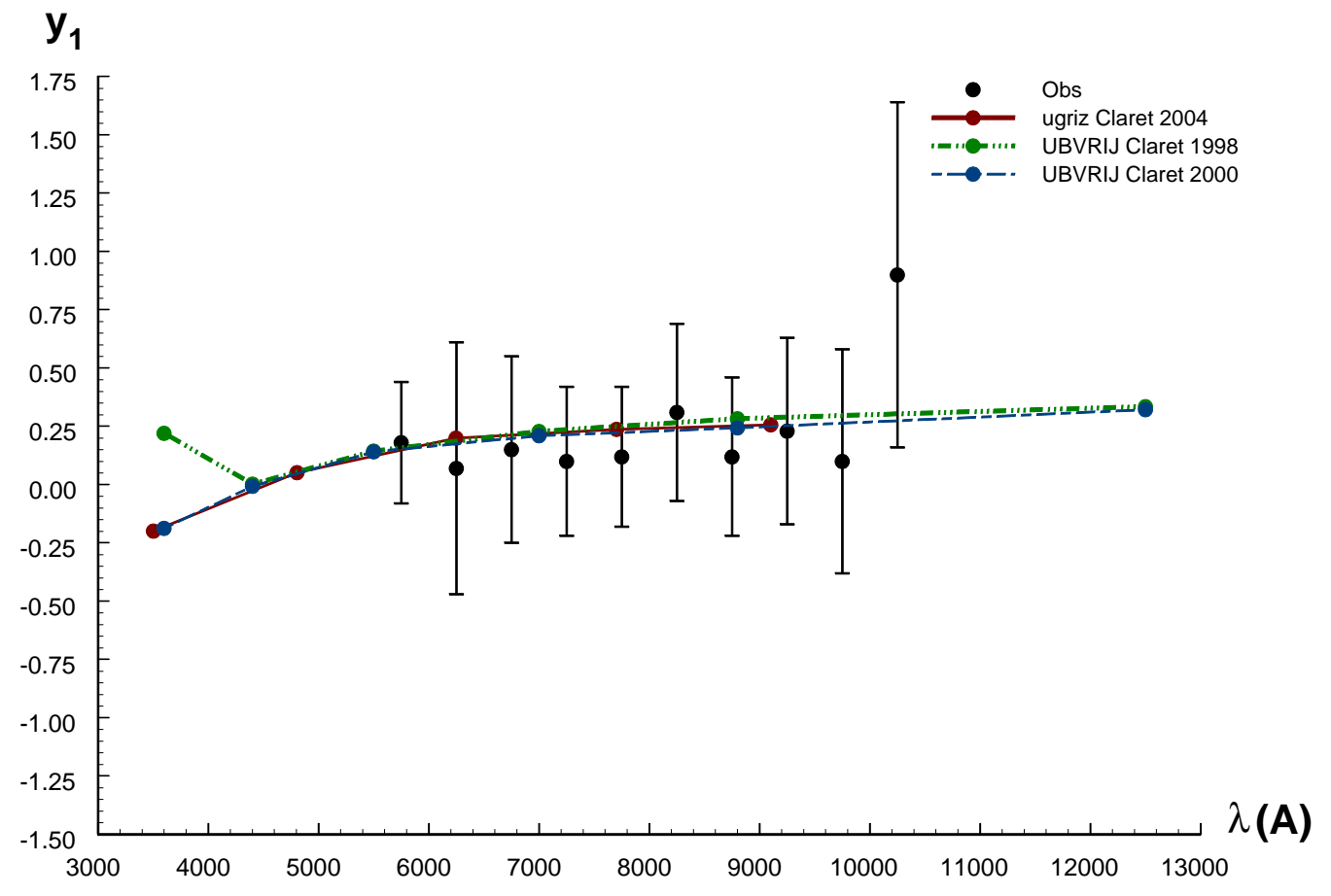

Fig 11: Same function as in Fig.8 but for the quadratic coefficient $y_{1}$. 


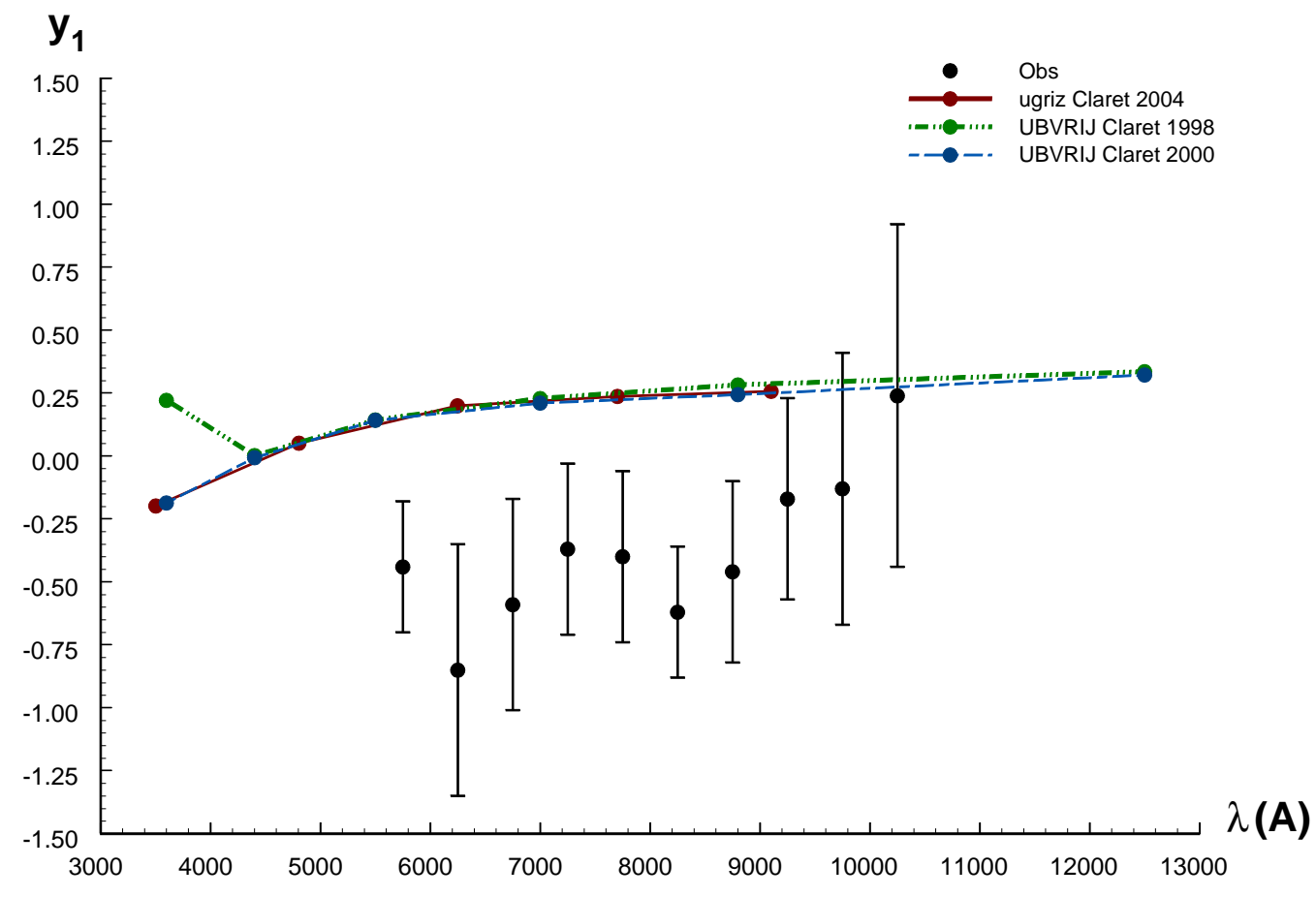

Fig 12: Same function as in Fig.11 but obtained for the analysis of the right branches of the light curves. 


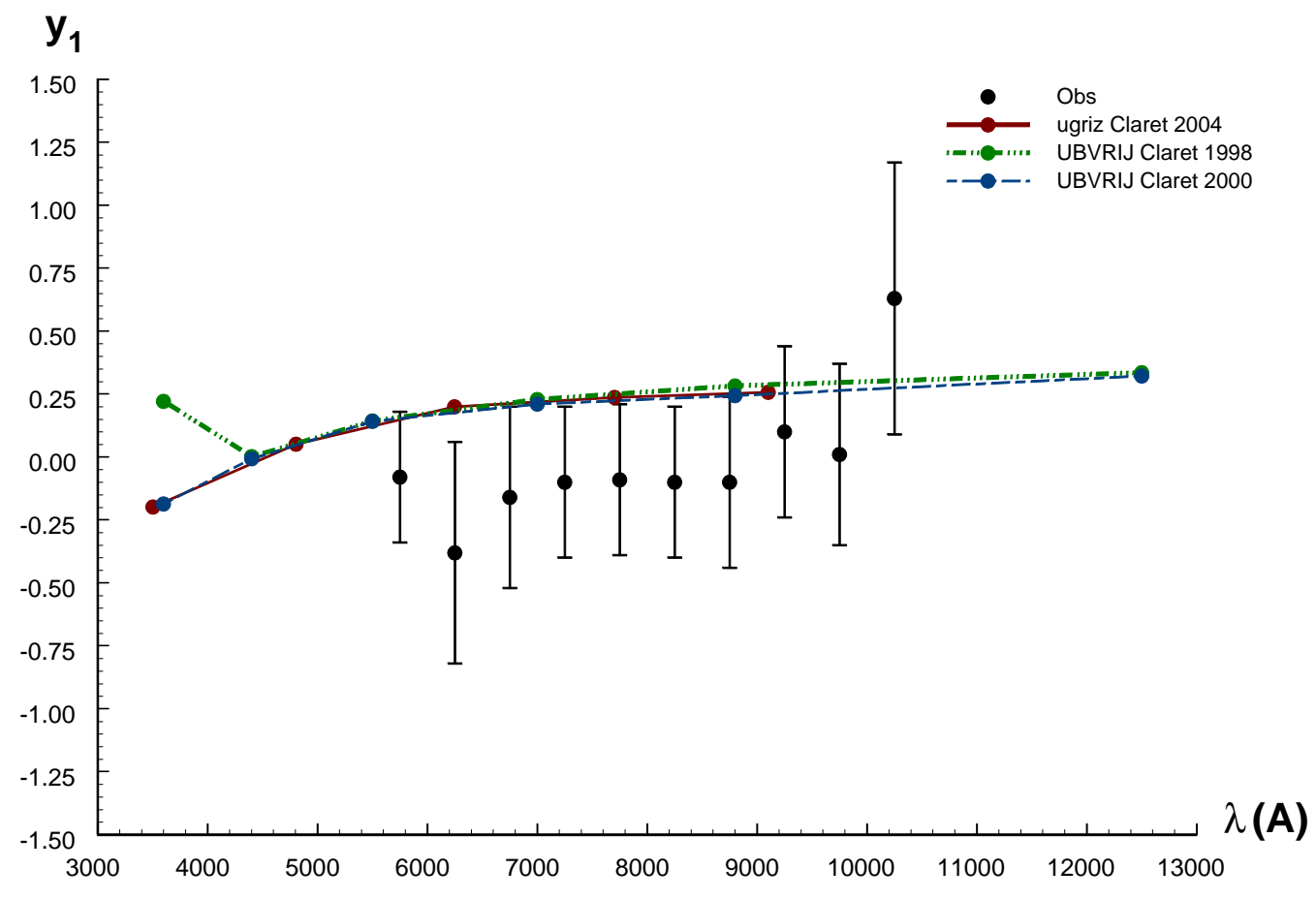

Fig 13: Same function as in Fig. 11 but obtained for the joint analysis of the left and right branches of the light curves. 


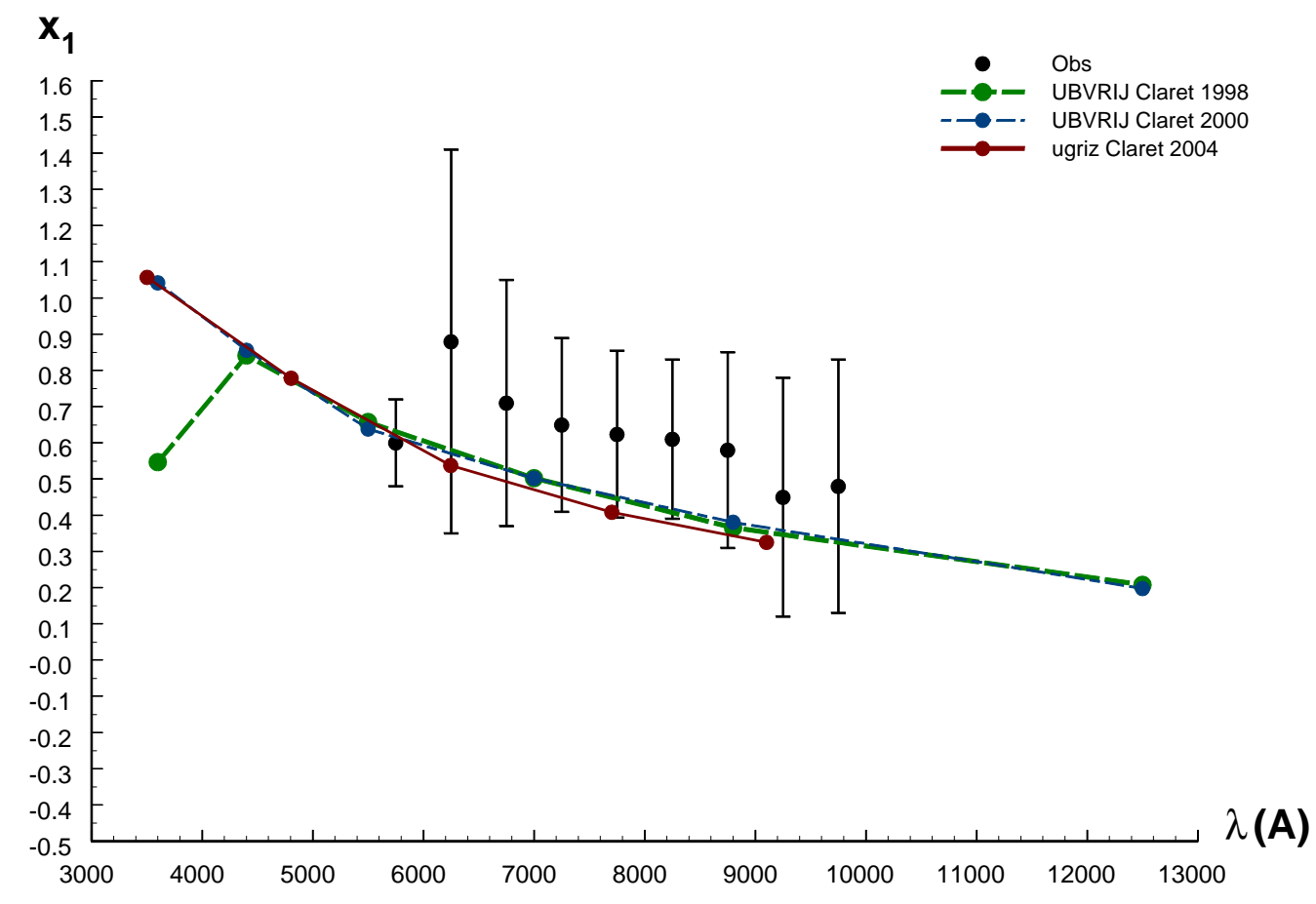

Fig 14: Same function as in Fig.10 but with the uncertainties in limb-darkening coefficients calculated using the confidencearea method based on the $\chi_{P}^{2}$ distribution; uncertainties correspond to $\gamma=0.955$. 


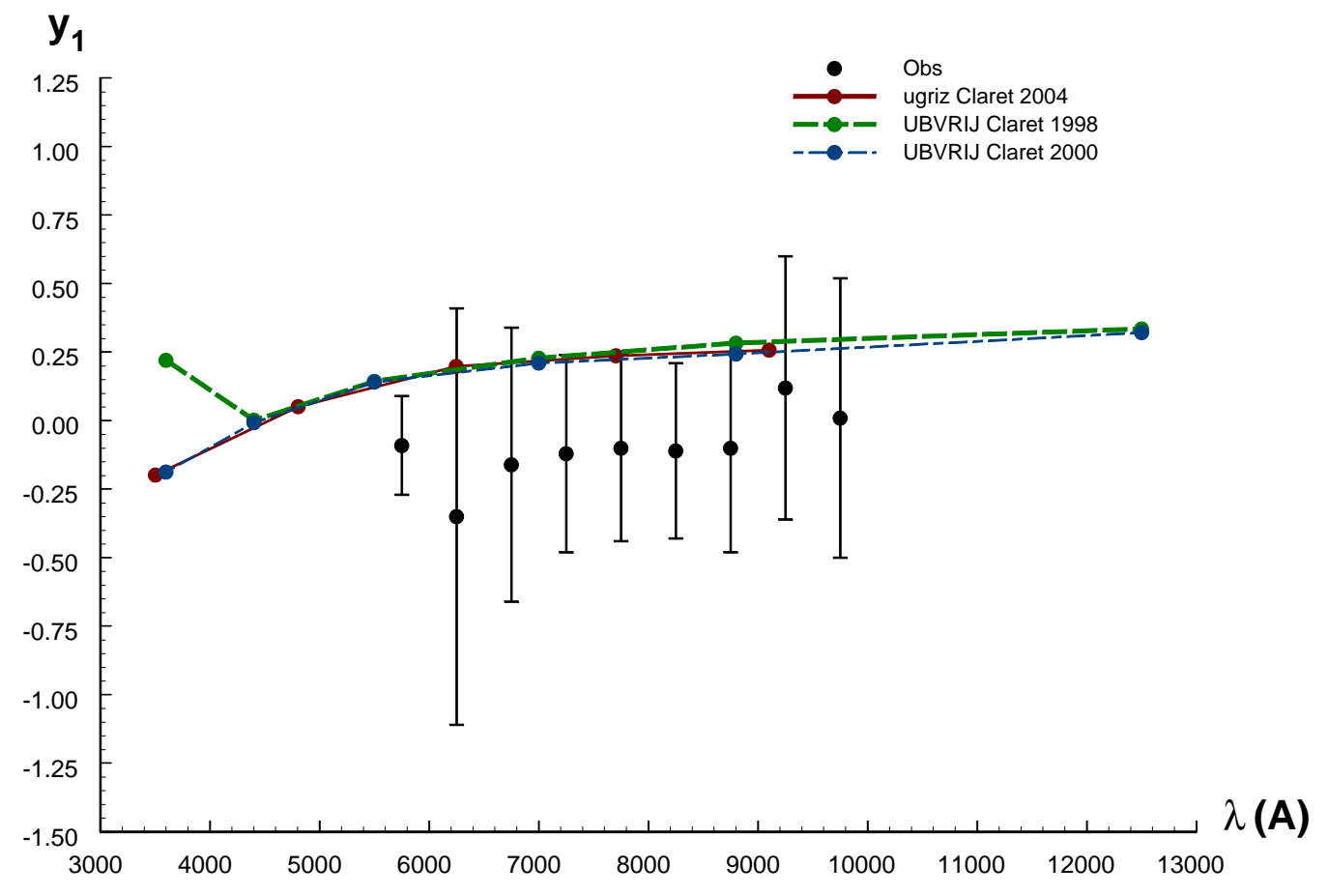

Fig 15: Same function as in Fig. 14 but for the quadratic coefficient $y_{1}$. 


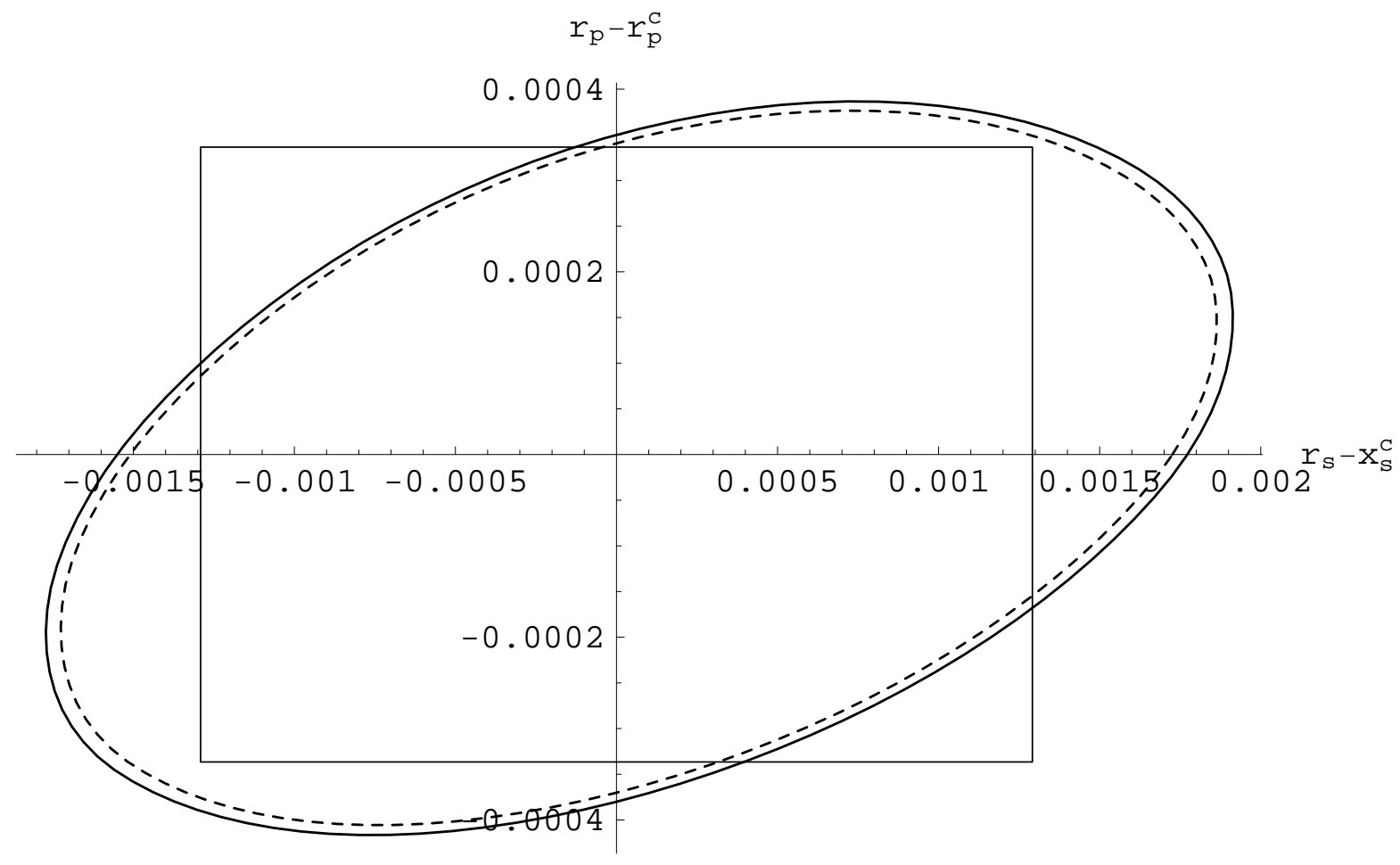

Fig 16: Projections of the confidence area (for $\gamma=0.955$ ) obtained using the $\chi_{P}^{2}$ (dashed line) and $\chi_{M}^{2}$ (solid line) distributions in the plane of the parameters $r_{s}, r_{p}$ when fitting the left branch of the light curve for $9500-10000 \AA$ with the quadratic limb-darkening law. The sides of the rectangle correspond to the $2 \sigma$ error intervals obtained using the differential-correction method. 


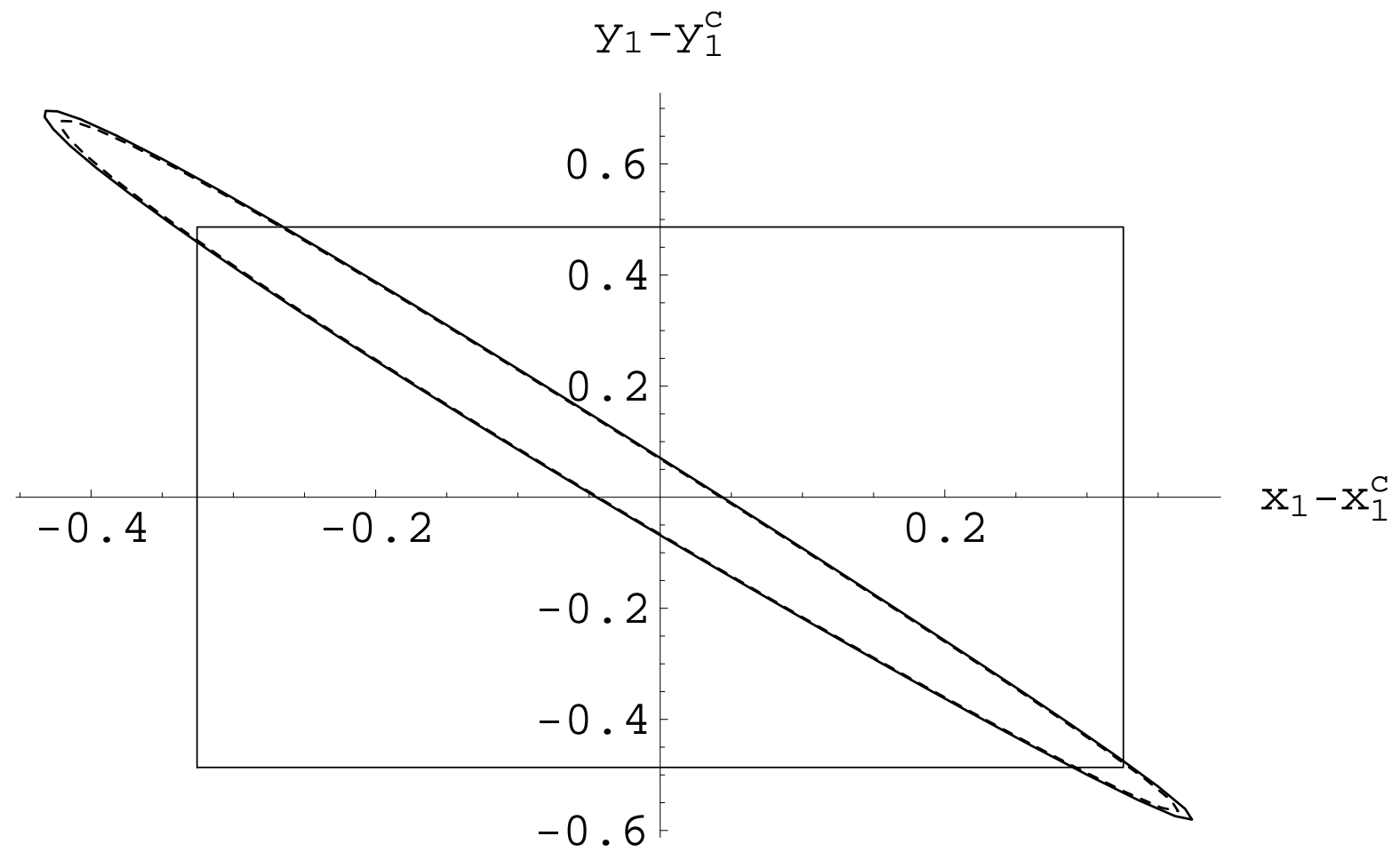

Fig 17: Projections of the confidence area (for $\gamma=0.955$ ) obtained using the $\chi_{P}^{2}$ (dashed line) and $\chi_{M}^{2}$ (solid line) distributions in the plane of the parameters $x_{1}, y_{1}$ when fitting the left branch of the light curve for $9500-10000 \AA$ with the quadratic limb-darkening law. The sides of the rectangle correspond to the $2 \sigma$ error intervals obtained using the differential-correction method. 